\title{
An Investigation of Transonic Resonance in a Mach 2.2 Round Convergent-Divergent Nozzle
}

\author{
Vance F. Dippold III ${ }^{1}$ \\ and \\ Khairul B. M. Q. Zaman² \\ NASA Glenn Research Center, Cleveland, Ohio 44135
}

\begin{abstract}
Hot-wire and acoustic measurements were taken for a round convergent nozzle and a round convergent-divergent (C-D) nozzle at a jet Mach number of 0.61 . The C-D nozzle had a design Mach number of 2.2. Compared to the convergent nozzle jet flow, the Mach 2.2 nozzle jet flow produced excess broadband noise (EBBN). It also produced a transonic resonance tone at $1200 \mathrm{~Hz}$. Computational simulations were performed for both nozzle flows. A steady Reynolds-Averaged Navier-Stokes simulation was performed for the convergent nozzle jet flow. For the Mach 2.2 nozzle flow, a steady RANS simulation, an unsteady RANS (URANS) simulation, and an unsteady Detached Eddy Simulation (DES) were performed. The RANS simulation of the convergent nozzle showed good agreement with the hot-wire velocity and turbulence measurements, though the decay of the potential core was over-predicted. The RANS simulation of the Mach 2.2 nozzle showed poor agreement with the experimental data, and more closely resembled an ideally-expanded jet. The URANS simulation also showed qualitative agreement with the hot-wire data, but predicted a transonic resonance at $1145 \mathrm{~Hz}$. The DES showed good agreement with the hot-wire velocity and turbulence data. The DES also produced a transonic tone at $1135 \mathrm{~Hz}$. The DES solution showed that the destabilization of the shock-induced separation region inside the nozzle produced increased levels of turbulence intensity. This is likely the source of the EBBN.
\end{abstract}

\section{Nomenclature}

$D_{j e t} \quad=\quad$ Nozzle exit diameter

$i=$ Index of data sample

$k=$ Turbulent kinetic energy

$M_{\text {jet }} \quad=$ Mach number for ideally-expanded jet

$n=$ Total number of data samples

$p \quad=\quad$ Static pressure

$r \quad=$ Radial coordinate; $\sqrt{y^{2}+z^{2}}$

$u, v, w=$ Components of velocity in the $x-, y$-, and $z$-directions

$u^{\prime} / U_{j e t}=$ Turbulence intensity in streamwise $(x-)$ direction, computed from $u_{R M S}$

$U_{j e t}=$ Velocity for ideally-expanded jet

$x, y, z=$ Orthogonal coordinate system

$\theta=$ Polar observation angle, measured from downstream axis

Subscripts

$\operatorname{avg}=$ Time-average value

RMS $=$ Root mean square value

$\infty \quad=$ Freestream value

${ }_{1}^{1}$ Aerospace Engineer, Inlets and Nozzles Branch, 21000 Brookpark Rd, and AIAA Senior Member

2 Aerospace Engineer, Inlets and Nozzles Branch, 21000 Brookpark Rd, and AIAA Associate Fellow 


\section{Introduction}

Convergent-divergent (C-D) nozzles, when operated at over-expanded nozzle pressure ratios, are known to produce significantly more noise than a convergent nozzle operating at the same conditions. This phenomenon is known as excess broadband noise (EBBN) ${ }^{1}$. Furthermore, at certain over-expanded nozzle pressure ratios C-D nozzles can produce resonances involving loud tones, referred to as transonic tones. Although these transonic resonances have been studied before ${ }^{1-3}$, they are not well understood.

A shortcoming of many experimental procedures for jet flows is that they do not allow the flow inside the nozzle to be investigated with great detail. Numerical simulations, though they have shortcomings of their own (e.g., cost, time, turbulence prediction and modeling), can easily show the flow inside the nozzle, in great detail.

Single-component velocity and turbulence data were collected for a round Mach 2.2 C-D nozzle at a pressure ratio that produced a transonic resonance, corresponding to an ideally-expanded jet Mach number, $M_{j e t}$, of 0.61 . These data were compared to similar data collected for a baseline nozzle (a round convergent nozzle) which involved no resonance or tones. Steady and unsteady numerical simulations were conducted for the Mach 2.2 nozzle using the Wind-US computational fluid dynamics code using Reynolds-Averaged Navier-Stokes (RANS) mode, Unsteady Reynolds-Averaged Navier-Stokes (URANS) mode, and Detached Eddy Simulation (DES) mode. Since the baseline convergent nozzle did not exhibit unsteady qualities, only RANS simulations were conducted for it. The results for the three computational fluid dynamics (CFD) simulations were compared to the experimental results. An objective of this paper is to shed light on the mechanisms that cause transonic tones using experimental measurements coupled with computational simulations.

\section{Experimental Methods}

All experimental measurements were made in an open jet facility (CW-17) at NASA Glenn Research Center. The facility consists of a 30-inch diameter plenum with flow conditioning units, through which compressed air flows before exhausting through an attached nozzle into the quiescent ambient air in the test chamber. The Mach 1.0 convergent nozzle and Mach 2.2 convergent-divergent (C-D) nozzle are two of a series of six round nozzles used in earlier studies ${ }^{1,4}$. Throughout this report, these nozzles will be referred to as the "M10T" and "M22" nozzles, respectively. Both nozzles have an exit diameter, $D_{j e t}$, of 2.0 inches and a total length of 7.5 inches. The M10T nozzle included a trip ring to ensure that the boundary layer at the exit was turbulent. The trip ring was located 1.689 inches upstream of the nozzle exit and was 0.125 inches wide. The upstream edge of the trip ring was mounted flush with the nozzle surface; the downstream edge consisted of a backstep that was 0.017 inches high. The divergent portion of the M22 C-D nozzle was designed using the method of characteristics. For the measurements taken for this study, the M22 nozzle did not include any boundary layer trips.

Single-component hot-wire measurements were performed using a TSI 1260-A10 probe. Data were sampled as the hot-wire probe was traversed through the jet plume along the following paths:

- $\quad$ axially along the jet plume centerline;

- radially through the plume at ten axial locations.

At each probe location, 20,000 data samples were collected, at a sampling rate ranging from $5000 \mathrm{~Hz}$ near the nozzle to $1250 \mathrm{~Hz}$ downstream from the nozzle. The hot-wire data were processed, such that average velocity, $u_{a v g}$, and turbulence intensity, $u^{\prime} / u_{j e t}$, were obtained. Acoustic measurements were also made for the jet flow. As the CW-17 open jet facility is semi-anechoic, limited acoustic measurements were performed using a single microphone located at $90^{\circ}$ with respect to the jet axis.

\section{Hot-wire and Acoustic Measurements}

Velocity and turbulence data were collected for the M10T and M22 nozzles for jet Mach number, $M_{j e t}$, equal to 0.61 . The average velocity, $u_{a v g}$, and the turbulence intensity, $u^{\prime} / u_{j e t}$, were calculated from the time-history of velocity, involving $n$ samples:

$$
u_{\text {avg }}=\left(\frac{1}{n}\right) \times \sum_{i=1}^{n} u_{i}
$$




$$
u^{\prime}=u_{R M S}=\sqrt{\left(\left(\frac{1}{n}\right) \times \sum_{i=1}^{n}\left(u_{i}\right)^{2}\right)-\left(u_{\text {avg }}\right)^{2}}
$$

$U_{j e t}$ is ideally-expanded jet velocity, computed from the nozzle pressure ratio. For the conditions during testing, $U_{j e t}$ was nominally $663.9 \mathrm{ft} / \mathrm{s}$. The average velocity and turbulence intensity are plotted along the centerline for the M22 and M10T nozzles in Figure 1. There is a significant difference in the behaviors of the plume for these two jets. The average velocity of the M10T nozzle shows that its potential core has a constant velocity nearly equal to the ideal jet velocity, $U_{j e t}$, for five to six jet diameters downstream of the nozzle. The turbulence intensity along the centerline is relatively low, but increases to a maximum value of $11.7 \%$ in the region where the jet potential core breaks down, $5^{*} D_{\text {jet }}$ to $6^{*} D_{\text {jet }}$ downstream of the nozzle exit. This is expected for a convergent nozzle jet flow. The average velocity of the M22 nozzle shows that the over-expanded jet exits the nozzle with a velocity of only about $85 \%$ of $U_{j e t}$ and continues to decrease through the jet plume. The turbulence intensity of the M22 nozzle jet is $17.5 \%$ just downstream of the nozzle exit - much larger than that of the M10T nozzle jet flow - and decreases as the flow progresses downstream.

Acoustic measurements were also taken for the M10T and M22 nozzle flows; the noise spectra for each nozzle are plotted in Figure 2. It is observed that the M22 nozzle flow has broadband noise levels that are 10 to $20 \mathrm{~dB}$ larger than those for the M10T nozzle flow. This is what is referred to as excess broadband noise (EBBN). It has been observed to be a problem with convergent-divergent nozzles operating at over-expanded conditions ${ }^{1}$. A second observation made from the noise spectra plot is that the M22 nozzle flow has peaks, beginning at about $1200 \mathrm{~Hz}$, and repeating at harmonics (e.g., $2400 \mathrm{~Hz}$, $3600 \mathrm{~Hz}$, etc.). These peaks represent the transonic tone. The transonic tone overshadows the EBBN by roughly $30 \mathrm{~dB}$. Therefore, for the same flow conditions, the M22 nozzle flow is much louder than the M10T nozzle flow.

\section{Numerical Methods}

\section{A. Wind-US Flow Solver}

Wind-US, version 35,6, was used to run computational fluid dynamics (CFD) simulations of the M10T and M22 nozzle flows. The Wind-US code is a general-purpose, Reynolds-Averaged Navier-Stokes (RANS) CFD solver for both structured and unstructured grids. The code is developed and managed by the NPARC Alliance, a partnership between NASA Glenn Research Center, USAF Arnold Engineering Development Complex, and The Boeing Company. The Wind-US code offers several numerical schemes, as well as several zero-, one-, and two-Equation turbulence models. A second-order Roe numerical scheme was used for all current simulations. The Menter Shear Stress Transport (SST) turbulence model ${ }^{7}$ was used for the RANS and unsteady RANS (URANS) simulations. The Spalart Detached Eddy Simulation (DES) model $^{8}$ - a hybrid scheme combining the Spalart-Allmaras single-Equation turbulence model and Large Eddy Simulation (LES) - was also used for one M22 nozzle simulation.

The trip ring installed in the M10T nozzle forced the boundary layer to transition from laminar to turbulent. It is typical to run numerical simulations of nozzle flows fully turbulent. To model the effect of the trip ring - forcing the boundary layer to transition from laminar to turbulent - the simulation was run laminar in a region near the wall upstream of the trip location. Everywhere else - including the region near the wall downstream of the trip ring location - the simulation was run using the Menter SST turbulence model.

For all simulations, the jet exhausted into a quiescent freestream, with a static temperature of $530^{\circ} \mathrm{R}$ and static pressure of 14.4 psi. The freestream Mach number was 0.01. It has been found that compressible flow solvers, including Wind-US, struggle with convergence for nozzle flows when the freestream velocity is set to zero; even a small freestream velocity helps the solver converge the solution more quickly. At the nozzle inflow, the total temperature was set to $530^{\circ} \mathrm{R}$; the total pressure was set to $18.51 \mathrm{psi}$, corresponding to a jet Mach number, $M_{j e t}$, of 0.61 . 


\section{B. Grid Strategy}

Structured grids were created using the commercially-available Pointwise ${ }^{\circledR}$ grid generation software$^{9}$. The M10T nozzle simulation used a 2-D, axisymmetric grid with 350,300 grid cells. The M10T nozzle grid is shown in Figure 3. The M22 nozzle RANS and URANS simulation used a 2-D, axisymmetric grid with 428,800 grid cells; the M22 nozzle DES used a 3-D grid with 91 million grid cells. (The decision to use only a 2-D, axisymmetric grid for the URANS simulation made the assumption that a URANS simulation using a 3-D grid solution would yield an axisymmetric solution. A future study into the validity of this assumption is highly recommended.) For all grids, the grid domain extended $10^{\star} D_{j e t}$ in the radial direction and $30^{*} D_{j e t}$ in the axial direction. Grid points were clustered radially towards the walls to ensure that the boundary layer was adequately resolved. The 3-D grid used an "H-grid" topology along the centerline (rather than an "O-grid" topology) in order to eliminate the singularity that would otherwise reduce the maximum global time step. The 3-D grid is pictured in Figure 4.

\section{Convergence}

The M22 nozzle RANS simulation used a Courant-Friedrichs-Levy (CFL) number of 0.5. The M10T nozzle RANS simulation was also initially set to use a CFL number of 0.5. However, the solution exhibited unsteadiness and it had to be converged using a constant time-step of $1.0 \mathrm{e}-7$ seconds. The URANS simulation used a global time step of 1.0e-7 seconds; the DES used a global time step of 5.0e-8 seconds. To speed up solution convergence, each simulation was sequenced through three levels of grids: first, the solution was solved using every fourth grid point (coarse), then using every second grid point (medium), and finally using every grid point (fine). Running the simulation on a sequence of grid resolutions allowed the overall flowfield to set up rather quickly on the coarser grids, before solving the turbulence details in the finest grid. This grid sequencing approach is standard practice for structured CFD calculations. Furthermore, grid sequencing allows an easy method of performing a grid convergence study. Figure 5 shows the velocity and turbulence intensity along the jet centerline of the M10T nozzle RANS simulation for three levels grid refinement (coarse, medium, and fine). While there are small differences between the solutions for the three grid levels, the solutions on the coarse and medium grid levels are monotonically converging upon the fine grid solution. Therefore, it can be concluded that the solution is essentially grid converged.

The convergence of each solution was tracked in several ways. The mass flow and thrust at the nozzle exit were monitored. While these quantities were not of primary interest to this study, nozzle mass flow and thrust quickly indicate if the jet flow is steady or unsteady, even indicating if resonant behaviors exist. The streamwise velocity and turbulent kinetic energy along the jet plume centerline were also monitored for solution convergence, as these were directly comparable to the data collected experimentally. Pressure and velocity were probed at several locations across the jet plume at $x / D_{j e t}=0.0$, 0.5 , and 1.0. These probed data were used to determine if resonant behavior existed in the jet flow. As mentioned earlier, the M10T nozzle RANS simulation exhibited some unsteady behavior when run using a constant CFL number, even though the experimental data showed the flow to be steady. This sort of behavior has been observed in previous studies ${ }^{10}$. The decision was made to run the simulation using a constant time-step of 1.0e-7 seconds. The solution converged within 50,000 constant time-step iterations (though, 200,000 constant time-step iterations were run) and showed no signs of unsteadiness. The M22 nozzle RANS simulation required 100,000 iterations to converge, using a constant CFL number of 0.5 . The M22 nozzle URANS simulation and DES required substantial iterations for the solutions to move through transients in the flowfield. After the solutions worked through the transients, statistical data were collected for one million constant time-step iterations for the URANS simulation and 1.58 million constant time-step iterations for the DES. All simulations were performed on the multi-node, multi-core NASA Advanced Supercomputing (NAS) Pleiades supercomputer ${ }^{11}$. 


\section{Numerical Results}

The results of the M10T nozzle and M22 nozzle simulations will be presented and compared to the experimental hot-wire data. Additionally, the M22 unsteady Reynolds-Averaged Navier-Stokes (URANS) and Detached Eddy Simulation (DES) results will be compared to acoustic measurements.

The average velocity, $u_{a v g}$, and turbulence intensity, $u^{\prime} / U_{j e t}$, were calculated for the unsteady numerical solutions (i.e., M22 nozzle URANS simulation and DES) using the time-history of velocity as in Equations 1 and 2. The steady solutions - M10T nozzle and M22 nozzle Reynolds-Averaged NavierStokes (RANS) simulations - already assume that the solution is an average solution in time. Therefore, the average velocity, $u_{\text {avg, }}$ is simply the $u$-component of velocity. When computing the turbulence intensity, $u^{\prime} / U_{j e t}$, from the RANS solutions, special considerations must be made. The turbulent kinetic energy, $k$, is one turbulent flow quantity computed by the RANS solution. In terms of the Reynolds stresses, the turbulent kinetic energy is defined as:

$$
k=\left(\frac{1}{2}\right) \times\left(\overline{u^{\prime 2}}+\overline{v^{\prime 2}}+\overline{w^{\prime 2}}\right)
$$

If it is assumed that the turbulence is isotropic (i.e., $\overline{u^{\prime 2}} \approx \overline{v^{\prime 2}} \approx \overline{w^{\prime 2}}$ ), turbulence intensity can be expressed as:

$$
u_{\text {isotropic }}^{\prime} \cong \sqrt{\left(\frac{2}{3}\right) \times k}
$$

However, it has been shown that turbulence is anisotropic for free shear layer flows, including jets ${ }^{12-15}$. Considering the data presented by DeBonis ${ }^{13}$ and Nallasamy ${ }^{15}$, the ratio of $\overline{u^{\prime 2}}: \overline{v^{\prime 2}}: \overline{w^{\prime 2}}$ is about 2:1:1 for a compressible round jet flow. Therefore, the turbulence intensity can be expressed as:

$$
u_{\text {jet data }}^{\prime} \cong \sqrt{k}
$$

The average velocity and turbulence intensity were compared along the jet centerline and along radial profiles through the jet plume. For the M10T nozzle flow, both expressions of turbulence intensity (Equations 4 and 5) were computed and compared.

The URANS simulation offers a unique opportunity, as it provides the ability to compare multiple methods of computing the turbulence intensity, $u^{\prime} / U_{j e t}$. Since the URANS was an unsteady simulation, it was natural to compute turbulence intensity according to Equation 2 using $u_{R M S}$. But, since the URANS used a turbulence model, it was also appropriate to compute turbulence intensity according to Equation 4 using $k_{a v g}$. For this discussion, the former method will be referred to as the "unsteady" method (Equation 2), as the value of $u_{R M S}$ comes from the unsteady statistics; the latter method (Equation 4) will be referred to as the "model" method, as the value of $k$ comes from the turbulence model. The consideration of these two methods begs to ask, which method of computing turbulence intensity is correct? The "model" method includes the turbulence information computed by the SST turbulence model; the "unsteady" method includes the actual turbulence statistics. Furthermore, could these methods be blended? One could sum together the respective portions of turbulent kinetic energy represented by the "model" and "unsteady" methods:

$$
u_{\text {sum }}^{\prime}=\sqrt{\overline{u^{\prime 2}}+\left(\frac{2}{3}\right) \times k_{\text {avg }}} \quad \text { Eq. } 6
$$

Unfortunately, this subject does not seem to have been addressed in great detail in literature. For the formal presentation of the numerical M22 nozzle solutions, the summed method in Equation 6 will be used to compute the turbulence intensity of the URANS simulation. There will be a separate discussion comparing the "model" and "unsteady" methods of computing turbulence intensity with experimental data and other numerical solutions.

\section{A. Mach 1.0 Nozzle}

A RANS simulation was completed for the baseline M10T nozzle. Figure 6 shows contour plots of the velocity and turbulence intensity. The average velocity and turbulence intensity along the centerline are compared for the RANS simulation and the experimental hot-wire data in Figure 7. The potential core of the RANS simulation is about $3.5^{*} D_{\text {jet }}$ longer than what was observed experimentally. Over-predicting the length of the jet potential core is a deficiency of RANS-based simulations and has been observed in other studies ${ }^{16}$. The delayed breakdown of the jet potential core by the RANS solution results in the peak 
turbulence intensity occurring $1.3^{\star} D_{\text {jet }}$ downstream of the location of the hot-wire peak turbulence intensity. The value of the peak turbulence intensity is dependent on the assumption used to compute it. Using the assumption that turbulence is isotropic (Equation 4), the peak turbulence intensity is within 1\% of the hot-wire data. The assumption used for anisotropic turbulence (Equation 5) simply scales the turbulence intensity by $22.5 \%$ (relative), showing that the RANS solution over-predicts the value of peak turbulence intensity. In Figure 8, radial profiles of the average velocity and turbulence intensity are compared through the jet plume. The RANS solution of the M10T nozzle jet shows good agreement with the hot-wire velocity data. The only disagreement is where the RANS solution over-predicts the length of the potential core, at $x / D_{j e t}=6$ and $x / D_{j e t}=8$. Again, the turbulence intensity is plotted using two different assumptions: isotropic turbulence (Equation 4) and anisotropic turbulence (Equation 5). The isotropic computation shows good agreement with the experimental data, only over-predicting the peak turbulence intensity from $x / D_{j e t}=0$ to $x / D_{j e t}=1$. Since the difference in the two computations of turbulence intensity is simply a scaling factor, the anisotropic computation of turbulence intensity consistently overpredicts the hot-wire data. Overall, the M10T RANS simulation showed good agreement with the experimental velocity data. The isotropic computation of turbulence - although it is based on less physical assumptions - allowed the RANS solution to show good agreement with the experimental turbulence intensity data. This may be a case in which a less physical assumption is right for the wrong reasons. The isotropic assumption (Equation 4) will be used for the remainder of this paper.

\section{B. Mach 2.2 Nozzle}

Three simulations were completed for the M22 nozzle: RANS, URANS, and DES. The RANS simulation used a constant Courant-Friedrichs-Levy (CFL) number, resulting in a steady-state solution. The RANS solution yielded average values of flow quantities (e.g., velocity, turbulent kinetic energy, and pressure), which could be compared to the average quantities of the unsteady solutions (i.e., URANS, DES). The URANS simulation completed 100 milliseconds of simulation time using a time step of 1.0e-7 seconds. The DES completed 79 milliseconds of simulation time using a time step of 5.0e-8 seconds. For the URANS simulation and the DES, the average data were calculated at a sampling rate of $100 \mathrm{kHz}$.

\section{Computational expenses}

Before progressing further, it is wise to pause and consider the computational expenses associated with these analyses. The RANS and URANS simulations used 10 computing cores on the NASA Advanced Supercomputing (NAS) Pleiades cluster. The DES used 300 computing cores on the NAS Pleiades cluster. The RANS simulation of the M22 nozzle required about 100 computational hours. The URANS simulation required about 1160 computational hours - greater than 11 times as much as the RANS simulation. The DES required about 485,500 computational hours - greater than 400 times as much as the URANS simulation. Because the DES used 300 computational cores, the simulation took on the order of two months of actual (or, wall clock) time to complete. The storage resources required were also significant: on the order of $400 \mathrm{MB}$ for the RANS simulation, $20 \mathrm{~GB}$ for the URANS simulation and 200 GB for the DES. For this study, as the fidelity of the simulation increased, the computational expenses increased even more so.

\section{Initial comparisons of jet plume with RANS, URANS, and DES solutions}

Figure 9 shows contours of the average velocity, $u_{\text {avg, }}$, along the $x-r$ (or, $x-y$ ) planes for all three M22 nozzle solutions. As expected from quasi-1-D nozzle flow theory, a shock is present just downstream of the nozzle throat. In all three M22 nozzle solutions, there is a small region of shock-induced separated flow just downstream of the shock. The flow does reattach in all three solutions, though the boundary layer is fairly thick as the flow exits the nozzle. The contour plots of average velocity also show that the RANS solution of the M22 nozzle sustains the jet potential core much longer than the two unsteady solutions. Figure 10 shows contours of the turbulence intensity, $u^{\prime} / U_{j e t}$, in the $x-r$ (or, $x-y$ ) planes. For the RANS solution, turbulence intensity is computed from turbulent kinetic energy, $k$, assuming isotropic turbulence (Equation 4); for the URANS solution, turbulence intensity is computed according to Equation 6. Qualitatively, the URANS and DES solutions look somewhat similar to each other; the RANS solution looks significantly different. The average velocity and turbulence intensity from the RANS 
solution for the M22 nozzle flow appear qualitatively similar to the solution of an ideally-expanded nozzle, like the M10T nozzle flow (refer to Figure 6a). While all three numerical solutions predict that the region of peak turbulence for this over-expanded jet flow occurs inside the nozzle at the shock-induced separation region, the amount of the turbulence intensity varies greatly. The unsteady solutions predict a larger amount of turbulence intensity, peaking at $34 \%$ and encompassing a region that nearly fills the divergent section of the nozzle and propagating $2 * D_{j e t}$ downstream of the nozzle exit. The RANS simulation predicts only a small region of turbulence intensity in the vicinity of the shock-induced separated flow, inside the nozzle. Though it may be difficult to see in Figure 10a, the turbulence intensity peaks at about $20 \%$ inside the nozzle. Downstream of the nozzle exit, the turbulence intensity is found in the jet plume shear layer. As will be shown later, the large region of turbulence intensity in the URANS simulation and DES is a result of the periodic destabilization of the separated flow inside the nozzle.

In Figure 11, the average velocity and turbulence intensity along the centerline of the M22 nozzle flow are plotted for the experiment and the three numerical solutions. The RANS solution shows no agreement with the experimental hot-wire data or the unsteady numerical solutions. Instead, the RANS solution does resemble an ideally-expanded jet solution (refer to the results of the M10T nozzle jet in Section V.A): the average velocity is equal to $U_{j e t}$ from the nozzle exit to $x / D_{j e t}=4.6$, beyond which the jet potential core breaks down and the jet velocity sharply decreases. This is further observed as the turbulence intensity sharply increases at $x / D_{\text {jet }}=4.6$, from $u^{\prime} / U_{j e t}=0 \%$ to $u^{\prime} / U_{j e t}=12 \%$. The URANS solution agrees with the experimental data at the nozzle exit, with an average velocity of $86 \%$ of $U_{j e t}$. However, downstream of the nozzle exit, the URANS solution shows little agreement with the hot-wire data. Instead of decreasing downstream of the nozzle exit, the jet velocity increases some, to an average velocity of $90 \%$ of $U_{j e t}$ at $x / D_{j e t}=2.8$, before steadily decreasing. The centerline turbulence intensity is at least $25 \%$ larger (relative) than what was observed experimentally, until about $x / D_{j e t}=3.5$. Downstream of $x / D_{j e t}=3.5$, the URANS solution shows reasonable agreement with the hot-wire data, though still overpredicts the hot-wire data (relative). The average velocity predicted by the DES shows the best agreement with the data, though not without its own deficiencies. The DES under-predicts the average velocity along the centerline by approximately $10-12 \%$ immediately downstream of the nozzle exit, to about $x / D_{j e t}=2$. Beyond $x / D_{j e t}=2$, the DES average velocity shows excellent agreement with the hot-wire data. The DES greatly over-predicts the turbulence intensity along the centerline for $x / D_{j e t}=0$ to $x / D_{j e t}=2$. There is again improved agreement downstream of $x / D_{\text {jet }}=2$ : the DES over-predicts turbulence intensity by no more than $20 \%$. Considering the over-prediction of turbulence intensity by the URANS simulation and DES in Figure 11, it may be inferred that the levels of turbulence intensity observed inside the nozzle in Figures $10 \mathrm{~b}$ and $10 \mathrm{c}$ may also be over-predicted.

\section{Comparison of methods for computing URANS turbulence intensity}

As discussed earlier, several methods were used to compute turbulence intensity for the URANS simulation of the M22 nozzle flow. The summed method (Equation 6) for computing turbulence intensity was presented in Figures 10 and 11. Contour plots of turbulence intensity for the "model" method (Equation 4) and "unsteady" method (Equation 2) are presented in Figure 12. Comparing these plots with those presented in Figure 10, the "model" method more closely resembles the RANS solution. The "unsteady" method resembles the turbulence field of the DES solution, but only near the nozzle $\left(x / D_{j e t}<2\right)$. The centerline turbulence intensity for the "unsteady" and "model" methods is compared in Figure 13. The turbulence intensity computed from the URANS "model" method resembles that of the RANS solution, shifted $2^{*} D_{j e t}$ upstream and with peak $u^{\prime}$ about $8 \%$ smaller (relative). This agrees with the shorter potential core length in the URANS solution (refer to Figure 9b). The URANS "unsteady" method over-predicts the turbulence intensity for $x / D_{j e t} \leq 3$, but it drops to zero downstream of that. The summed method of computing the URANS turbulence intensity tracks those of the "model" and "unsteady" methods and smoothly transitions between them from $x / D_{\text {jet }}=3$ to $x / D_{\text {jet }}=5$. This is because the summed method incorporates both the portion of turbulence intensity from the unsteady velocity field and the portion of turbulence intensity from the RANS turbulence model. The summed turbulence intensity shows much better qualitative agreement with the DES solution and the experimental data than either the 
"model" or "unsteady" methods, alone. This topic could be the subject of its own study. For this effort, it has been shown that it is necessary to consider both portions of turbulence for an URANS simulation.

\section{Further analysis of the jet plume with RANS, URANS, and DES solutions}

Returning to the comparison of the RANS, URANS, and DES solutions for the M22 nozzles, Figure 14 shows the average velocity and turbulence intensity plotted for the three numerical solutions and hot-wire data along lines extending radially from the jet centerline at various axial locations in the jet plume. Recalling the large differences between the RANS solution and the hot-wire data along the centerline, it is no surprise that there is very little agreement between the RANS solution and the hot-wire data through the plume at any locations. The RANS solution shows qualitative agreement with the hotwire data only at the outer fringes of the jet plume $\left(r / D_{j e t} \geq 1\right)$ at the downstream locations $\left(x / D_{j e t} \geq 10\right)$, and only for the average velocity. At the nozzle exit, the average velocity of the URANS solution shows good agreement with the hot-wire data for $r / D_{j e t} \leq 0.4$. Downstream of the nozzle exit, the URANS solution overpredicts the average velocity in the jet plume. The URANS turbulence intensity shows only qualitative agreement with the hot-wire data: it captures some of the trends observed in the hot-wire turbulence intensity data, but the values are over-predicted. The URANS turbulence intensity does show good agreement with the DES-predicted turbulence intensity at $x / D_{j e t}=0.5$. At $x / D_{j e t} \geq 4$, the URANS turbulence intensity is influenced by the RANS turbulence model rather than the unsteady velocity, as the URANS turbulence intensity more-closely resembles that of the RANS simulation. Compared to the hot-wire data, the DES consistently under-predicts the average velocity by no more than $10 \%$ in some regions $\left(x / D_{j e t} \leq 1\right.$ and $\left.x / D_{j e t} \geq 8\right)$. However in other regions $\left(2 \leq x / D_{j e t} \leq 6\right)$, the DES average velocity shows excellent agreement with the hot-wire data. These observations are consistent with the centerline average velocity plotted in Figure 11. The DES turbulence intensity shows good agreement with the hot-wire data for $x / D_{j e t}>2.0$. Closer to the nozzle exit, the DES turbulence intensity is much larger than the experimental data, again consistent with Figure 11. Four lines are shown for the DES in Figure 11, showing the solution plotted along $90^{\circ}$ azimuthal intervals. The differences in average velocity and turbulence intensity at the different angles show that the flow is not symmetric. The instantaneous flow is highly 3-D, but over time the differences in $u_{\text {avg }}$ and $u^{\prime} / \iota_{j e t}$ at these four angles decreased. It can be inferred that if the DES is run for enough cycles, the statistics (i.e, $u_{\text {avg }}$ and $u^{\prime} / U_{j e t}$ ) will show that the flow is axisymmetric.

It is curious why the numerical simulations did a poor job predicting the flow - especially the turbulence intensity - a short distance downstream of the nozzle (i.e., $x / D_{j e t}<2$ ). One possible cause could be that the inflow conditions are not being modeled sufficiently. Unfortunately, there were limited means to interrogate the nozzle inflow in the open jet facility. Without knowing the experimental nozzle inflow conditions - such as radial velocity and turbulence intensity - attempts to model the flow with greater accuracy will not necessarily help in reducing the uncertainty. Resolving these discrepancies could be the focus of a future study.

\section{Acoustic predictions using unsteady simulations}

The average and root mean square (RMS) value of pressure along the centerline are shown for the flow inside the nozzle in Figure 15 for the numerical solutions. There is modest agreement between the unsteady solutions, which predict the shock (represented by the sharp increase in $p_{\text {avg }}$ following the minimum $\left.p_{\text {avg }}\right)$ at $x / D_{j e t}=-1.45$. The RANS solution predicts the shock to be upstream of the location predicted by the unsteady solutions, at $x / D_{j e t}=-1.56$. From the unsteady simulations, it was possible to compute the RMS pressure, $p_{R M S}$. The single peaks along the URANS and DES plots of $p_{R M S}$ indicate that resonance is present in each of these simulations. The transonic resonance (spectral data shown later) is inferred to be in the fundamental mode involving a one quarter standing wave within the divergent section ${ }^{2}$.

Unsteady data were collected for a dozen points from the URANS simulation and the DES. The time history of the unsteady pressure for several of these probe points is plotted in Figure 16 for $100 \mathrm{~ms}$ of URANS simulation time and $79 \mathrm{~ms}$ of DES simulation time. Power spectral density (PSD) calculations were performed on the unsteady static pressure for each of these unsteady numerical solutions; the results are plotted in Figure 17. Recall that the experimental sound pressure level (SPL) spectrum for the M22 nozzle at $M_{j e t}=0.61$ was presented earlier, in Figure 2 . The peak at $1200 \mathrm{~Hz}$ in Figure 2 represents the 
transonic resonance tone; the subsequent peaks represent harmonics. In a similar manner, the sharp spikes in the PSD plot in Figure 17 indicate frequencies of the resonant tones and harmonics. The URANS simulation has a resonance tone of $1145 \mathrm{~Hz}$, with harmonics at $2288 \mathrm{~Hz}, 3434 \mathrm{~Hz}, 4578 \mathrm{~Hz}$, etc. The DES has a resonant tone of $1135 \mathrm{~Hz}$, with harmonics at $2270 \mathrm{~Hz}, 3415 \mathrm{~Hz}, 5689 \mathrm{~Hz}$, etc. The URANS simulation and DES both predicted the resonant frequency within about $5.5 \%$ of the experimentally measured transonic resonance tone.

\section{Investigating the resonant behavior with DES}

An advantage offered by CFD simulations is that they can be used to investigate regions of flow obscured during experimental testing. Figure 18 is an attempt to deconstruct the M22 nozzle's internal flow during the transonic resonance using the DES. Figure 18 attempts to show six stages observed in resonant jet flow. At each stage, three contour plots from the DES are pictured: instantaneous numerical shadowgraph (computed using the gradient of density); instantaneous axial velocity, $u / U_{j e t}$; and instantaneous static pressure, $p / p_{\infty}$. The following stages describe the flow:

1. Inside the nozzle, a shock wave is located just downstream of the throat. There is a small region of shock-induced separated flow, but the flow quickly reattaches to the nozzle wall (indicated by arrow).

2. The shock wave moves upstream and a series of oblique shock and expansion waves form (indicated by arrow). The separation point inside the nozzle moves upstream and the separation region grows.

3. The shock train has moved upstream of the nozzle throat. The separation region destabilizes (indicated by arrow).

4. A plug of high-speed flow moves through the nozzle. A pressure wave moves through the plume ahead of the high speed flow (indicated by arrow).

5. As the plug of high speed flow exits the nozzle, the nozzle exit begins to re-pressurize (indicated by arrow). A shock wave reforms downstream of the throat.

6. A pressure wave propagates from the nozzle exit, both downstream through the plume and upstream into freestream (indicated by arrow). The shock wave near the nozzle throat changes structure and the shock-induced separation region grows.

It appears that the destabilization of the separated region on the nozzle internal surface triggers the resonant behavior. Returning to the contour plot of turbulence intensity in Figure 10c, it is observed that the region of flow near the separation region has the greatest level of turbulent intensity. Because noise is directly related to turbulence intensity, it can be inferred that this region of increased turbulence intensity leads to the excess broadband noise (EBBN) produced by the over-expanded nozzle.

\section{Conclusions}

Hot-wire and acoustic measurements were carried out for a round, convergent nozzle and a round, convergent-divergent (C-D) nozzle with a design Mach number of 2.2. Both nozzles were run with a jet Mach number of 0.61 in a semi-anechoic test cell. The over-expanded flow through the Mach 2.2 nozzle produced excess broadband noise (EBBN) and a transonic tone.

The steady Reynolds-Averaged Navier-Stokes (RANS) simulation of the round, convergent nozzle showed good agreement with the experimental hot-wire data, though the breakdown of the jet potential core was prolonged by approximately four jet diameters. This result is attributed to limitations in RANS turbulence models and is consistent with previous studies. Three numerical simulations were performed for the C-D nozzle: a steady RANS simulation, an unsteady RANS (URANS) simulation, and an unsteady Detached Eddy Simulation (DES). All three numerical solutions predicted a shock inside the nozzle, just downstream of the nozzle throat, and a region of shock-induced separated flow. In the timeaveraged solutions, the shock-induced separated flow reattached before the flow exited the nozzle. The RANS showed the least agreement with the experimental hot-wire data; it more closely resembled an ideally-expanded jet flow. The URANS simulation and DES both predicted resonant tones within $5.5 \%$ of the $1200 \mathrm{~Hz}$ tone observed in the acoustic measurements, at $1145 \mathrm{~Hz}$ and $1135 \mathrm{~Hz}$, respectively. The URANS solution showed only qualitative agreement with the hot-wire measurements. The DES solution 
showed good agreement. The latter solution under-predicted the average velocity by $10-12 \%$ immediately downstream of the nozzle, but the agreement was good beyond $x / D_{j e t}=2$. Downstream of $x / D_{j e t}=2$, the DES solution over-predicted the turbulence intensity in the jet plume, within $20 \%$ of the hot-wire measurement. Of the three numerical methods used to simulate the over-expanded Mach 2.2 C-D nozzle, the DES most closely matched the experimental measurements. Furthermore, the DES solution permitted an investigation of the flow structure inside the nozzle of a jet flow undergoing a resonant cycle. This has not been possible with experimental techniques, but it is a clear advantage of numerical simulations. The DES solution showed that the destabilization of the shock-induced separation region lead to increased levels of turbulence intensity, producing EBBN.

As the level of fidelity was increased with each simulation method (i.e., RANS, URANS, DES), the computational costs also increased. For the Mach 2.2 C-D nozzle, the RANS simulation failed to capture any unsteady effects and the solution resembled a very different nozzle jet flow. The URANS simulation performed better, as it captured - at least qualitatively - some of the velocity and turbulence structure inside the nozzle and in the first several nozzle diameters downstream and predicted a transonic resonance tone within 5.5\% of the acoustic measurements. It was imperative to consider both the unsteady and modeled portions of the URANS turbulence intensity, as considering only one or the other produced poor predictions of the turbulence. The cost the URANS solution was about 11 times that of the RANS solution, but it was still relatively inexpensive. The DES produced the best results, capturing much of the velocity and turbulence structure through the jet plume and also predicting the transonic resonance tone within 5.5\% of the acoustic measurements. However, the DES cost upwards of 400 times as much as the URANS simulation.

\section{Recommendations}

When conducting numerical simulations of most flows, the selection of method (e.g., RANS, URANS, DES) depends on the level of fidelity required for the solution. While a RANS simulation is appropriate for an axisymmetric, ideally-expanded, round, convergent nozzle, a RANS simulation is not appropriate for unsteady jet flows. If the jet flow is unknown, a strategy of increasing-fidelity simulations is recommended. Begin with an inexpensive RANS simulation. If the flow solution does not converge easily, that is a sign that the flow might be unsteady. Proceed to run a URANS simulation, which is still relatively inexpensive (at least for an axisymmetric jet flow). If the URANS simulation converges to a steady solution, then the flow is most likely steady. If the URANS simulation does not converge, shows signs of resonance, or converges to a different solution than the RANS simulation, then the flow is probably unsteady. If the solution is unsteady and greater fidelity is required, then a DES should be completed. For design purposes or for jet flows in which the structure of the jet is unknown, a series of increasing-fidelity, unsteady numerical simulations will likely be necessary to ascertain the level of EBBN and transonic tones that are produced in new nozzles.

There are still areas to explore. A future study could confirm these predictions for nozzle flows that produce EBBN, yet do not produce transonic tones. Additionally, investigations could be conducted into more realistic nozzles - including dual-stream, C-D nozzles - operated in over-expanded conditions. Finally, one could investigate whether it is possible to design C-D nozzles in such a way as to reduce EBBN, perhaps by trying to re-stabilize the shock-induced separation region.

\section{Acknowledgments}

This work was supported by the Fundamental Aeronautics Program's High-Speed Project. Author V. F. D. is grateful for the recommendations given by Nicholas Georgiadis. 


\section{References}

1 Zaman, K. B. M. Q., Bridges, J. E., Brown, C. A., "Excess Broadband Noise Observed with Overexpanded jets," AIAA Journal, Vol. 48, No. 1 (2010), pp. 202-214. doi: 10.2514/1.43383.

2 Loh, C. Y., Zaman, K. B. M. Q., "Numerical Investigation of Transonic Resonance with a Convergent-Divergent Nozzle," AIAA Journal, Vol. 40, No. 12 (2002), pp. 2393-2401. doi: 10.2514/2.1607.

3 Zaman, K. B. M. Q., Bencic, T. J., Clem, M. M., Fagan, A. F., “Shock-Induced Boundary Layer Separation in C-D Nozzles and Its Impact on Jet Noise," AIAA Paper 2011-1031, Jan. 2011.

4 Morris, P. J. and Zaman, K. B. M. Q., "Velocity measurements in jets with application to noise source modeling," Journal of Sound and Vibration (2009). doi: 10.1016/j.jsv.2009.09.024.

5 Towne, C. E., “Wind-US Users Guide, Version 2.0,” NASA/TM - 2009-215804, Oct. 2009.

6 Georgiadis, N. J., Yoder, D. A., Towne, C. S., Engblom, W. A., Bhagwandin, V. A., Power, G. D., Lankford, D. W., and Nelson, C. C., "Wind-US Code Physical Modeling Improvements to Complement Hypersonic Testing and Evaluation," AIAA Paper 2009-193, Jan. 2009.

7 Menter, F. R., "Two-Equation Eddy Viscosity Turbulence Models for Engineering Applications," AIAA Journal, Vol. 32, No. 8 (1994), pp. 1598-1605. doi: 10.2514/3.12149.

8 Spalart, P. R., "Young-Person's Guide to Detached-Eddy Simulation Grids," NASA/CR - 2001211032, July 2001.

9 Pointwise ${ }^{\circledR}$ [software package], Ver. 17.1 Release 4, Pointwise, Inc., Fort Worth, TX, 2013.

${ }^{10}$ Dippold, V. F., "Computational Simulations of Convergent Nozzles for the AIAA 1st Propulsion Aerodynamics Workshop," NASA/TM-2014-218329, Aug. 2014.

${ }^{11}$ NASA High-End Computing Capability [webpage], http://www.nas.nasa.gov/hecc/, [cited 1 November 2014].

12 Yoder, D. A., DeBonis, J. R., and Georgiadis, N. J., "Modeling of Turbulent Free Shear Flows," AIAA Paper 2013-2721, July 2013.

${ }^{13}$ DeBonis, J. R., "A High-Resolution Capability for Large-Eddy Simulation of Jet Flows," AIAA Paper 2010-5023, July 2010.

${ }^{14}$ Adumitroaie, V., Ristorcelli, J. R., Taulbee, D. B., "Progress in Favre-Reynolds stress closures for compressible flows," Physics of Fluids, Vol. 11, No. 9 (1999), pp. 2696-2719. doi: 10.1063/1.870130.

${ }^{15}$ Nallasamy, M., "Survey of Turbulence Models for the Computation of Turbulent Jet Flow and Noise," NASA/CR -1999-206592, March 1999.

${ }^{16}$ Georgiadis, N. J., Rumsey, C. L., Yoder, D. A., and Zaman, K. B. M. Q., "Turbulence Model Effects on Calculation of Lobed Nozzle Flowfields," Journal of Propulsion and Power, Vol. 22, No. 3 (2006), pp. 567-575. doi: 10.2514/1.17160. 


\section{Figures}

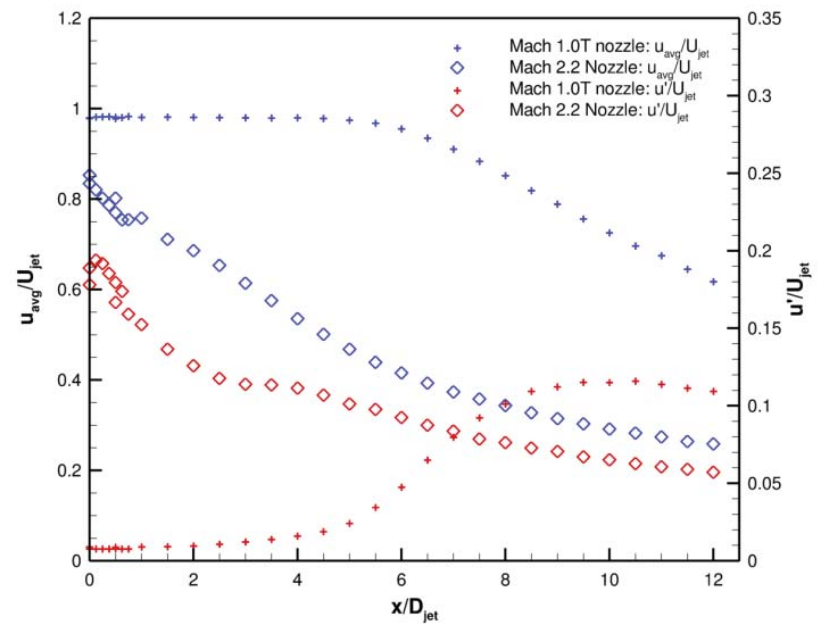

Figure 1: Experimental average velocity (blue) and turbulence intensity (red) along jet centerline for Mach 1.0T and Mach 2.2 nozzles flows at $M_{j e t}=0.61$.

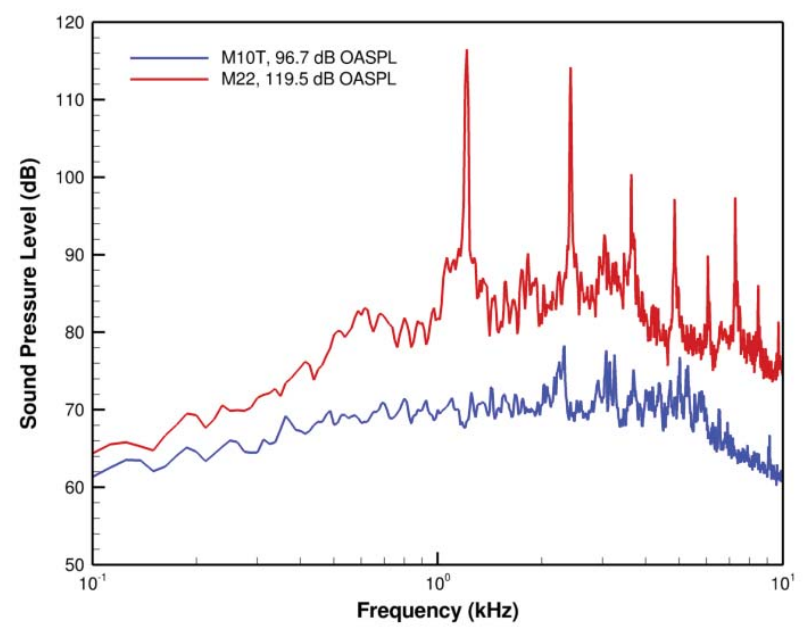

Figure 2: Experimental sound pressure level spectra at $\theta=90^{\circ}$ for the Mach 1.0T and Mach 2.2 nozzles at $M_{\text {jet }}=0.61$.

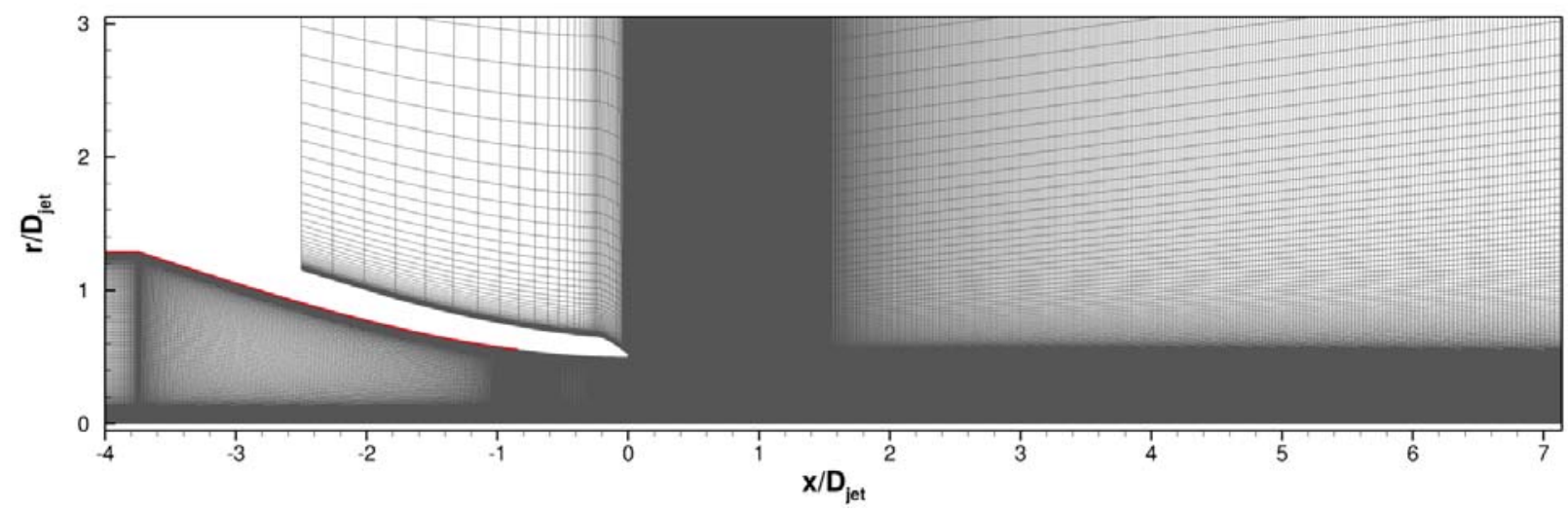

Figure 3: Mach 1.0T nozzle grid. Red indicates laminar regions, upstream of boundary layer trip. 


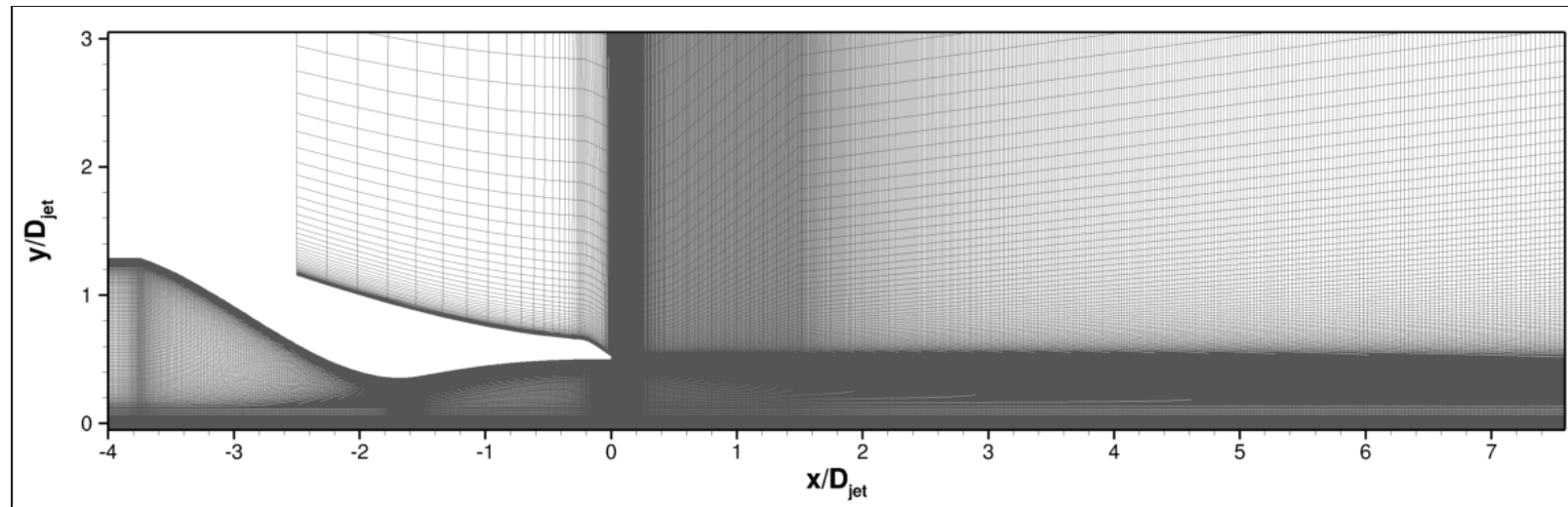

a) Grid along x-y plane.

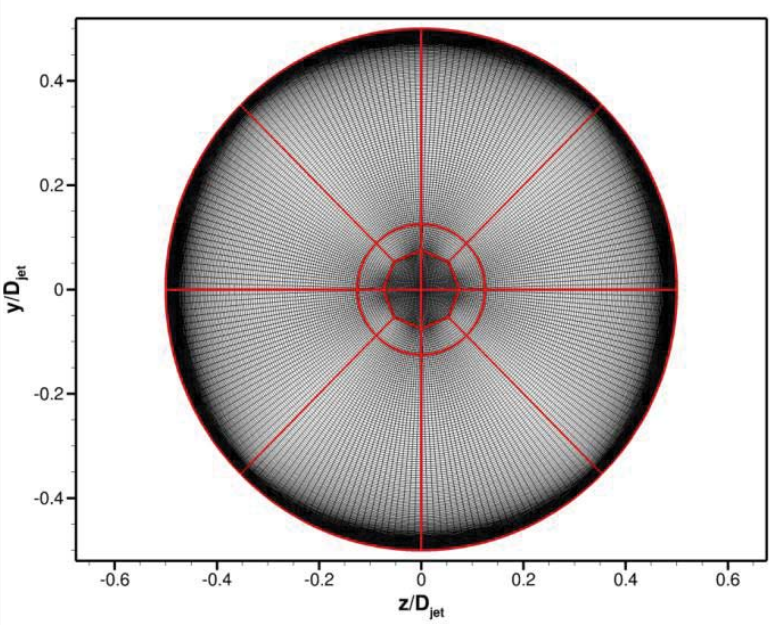

b) Grid at nozzle exit.

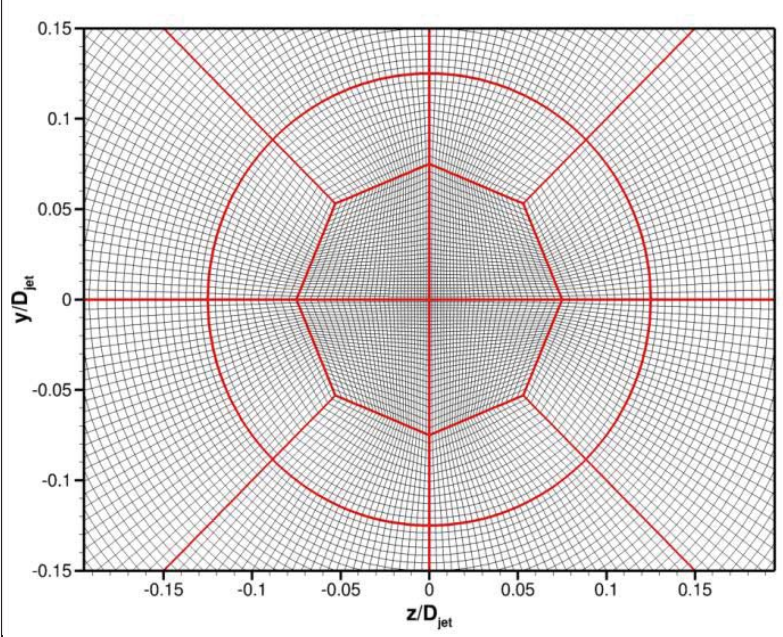

c) "H-grid" topology along centerline.

Figure 4: Mach 2.2 nozzle 3-D grid. The red lines in sub-figures $b$ and $c$ indicate zonal boundaries.

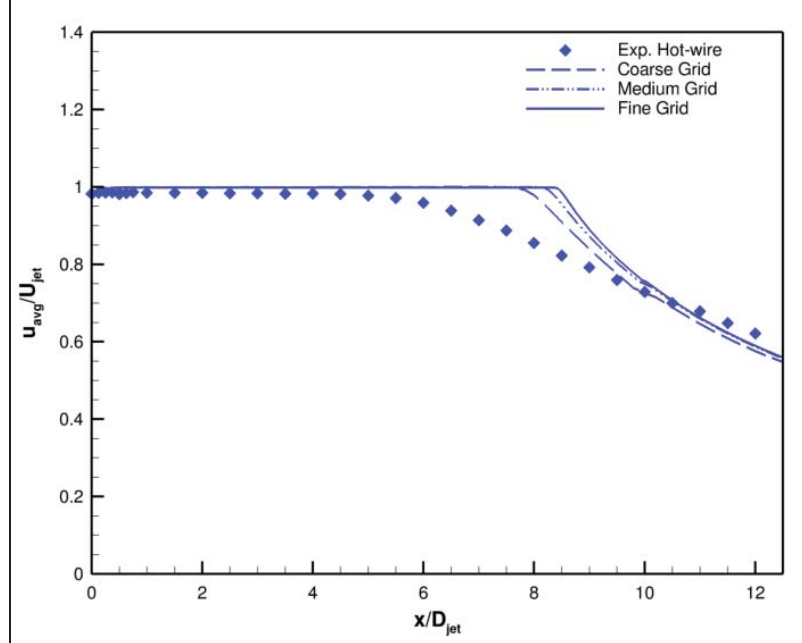

a) Average velocity.

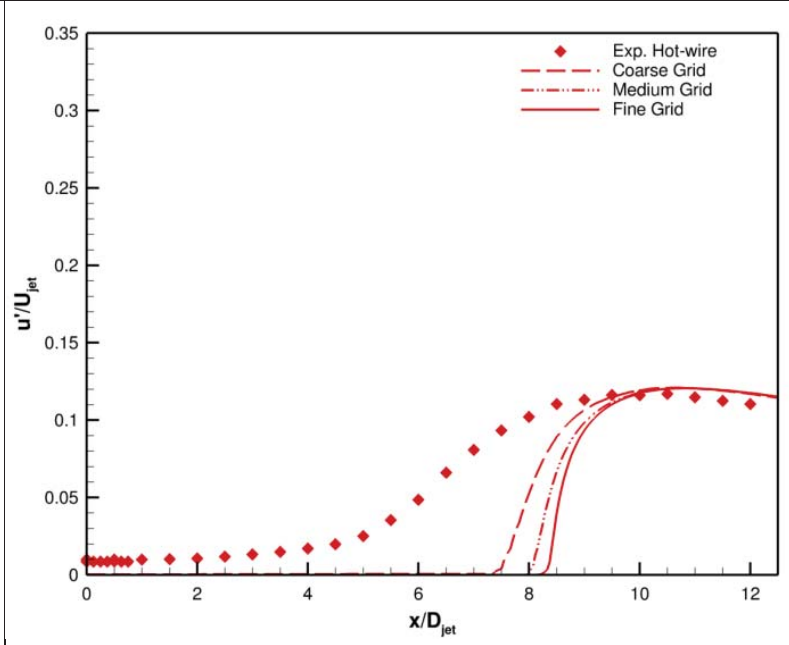

b) Turbulence intensity.

Figure 5: Grid convergence of M10T nozzle RANS simulation. Plots show average velocity (blue) and turbulence intensity (red) along jet centerline for three levels of grid refinement. Plots also include hot-wire data. 


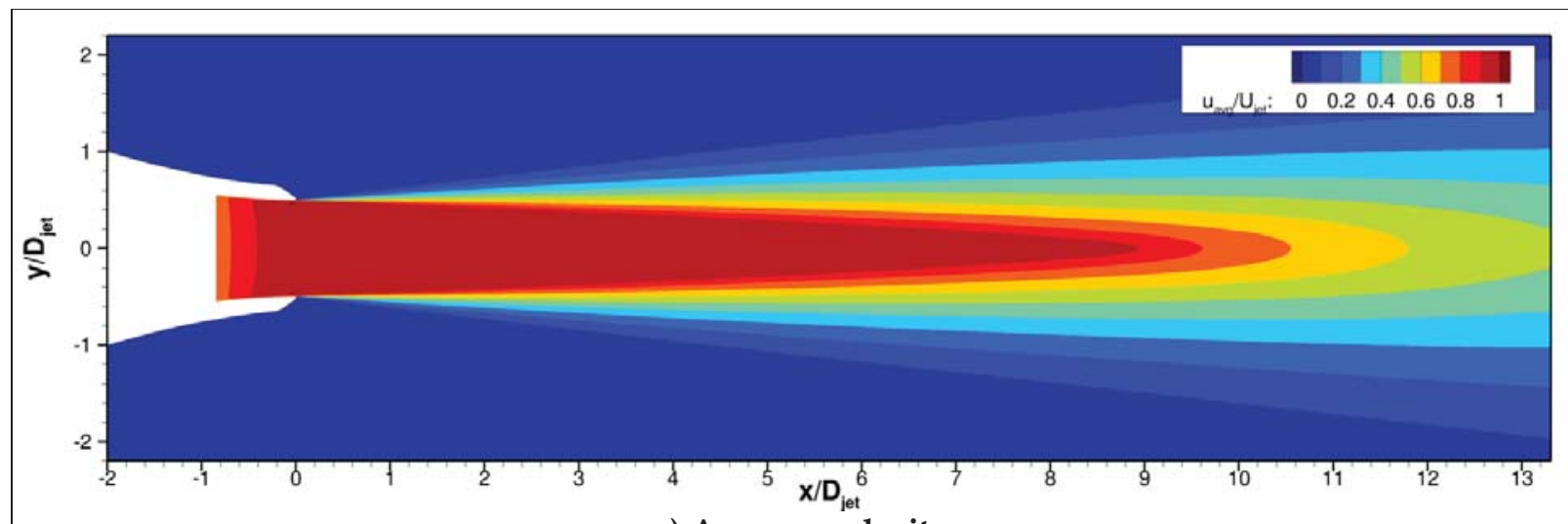

a) Average velocity.

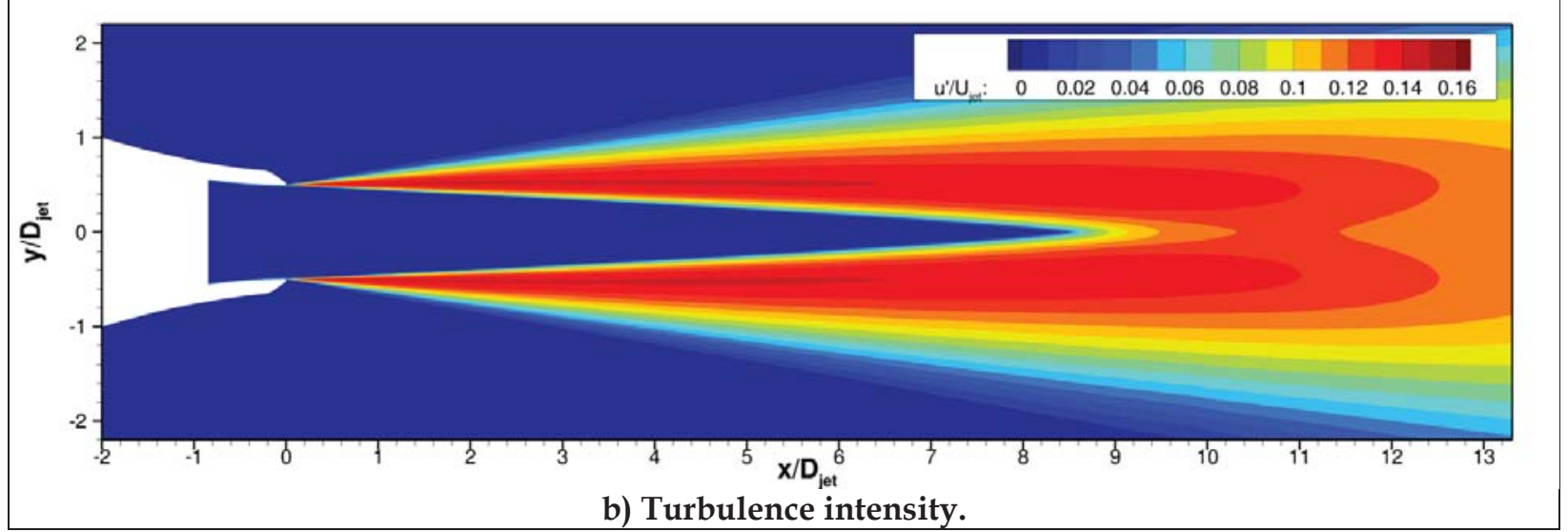

Figure 6: Contours of average velocity and turbulence intensity for the M10T nozzle at $M_{j e t}=0.61$.

Results from RANS simulation are shown. Turbulence intensity computed assuming isotropic turbulence (Equation 4).

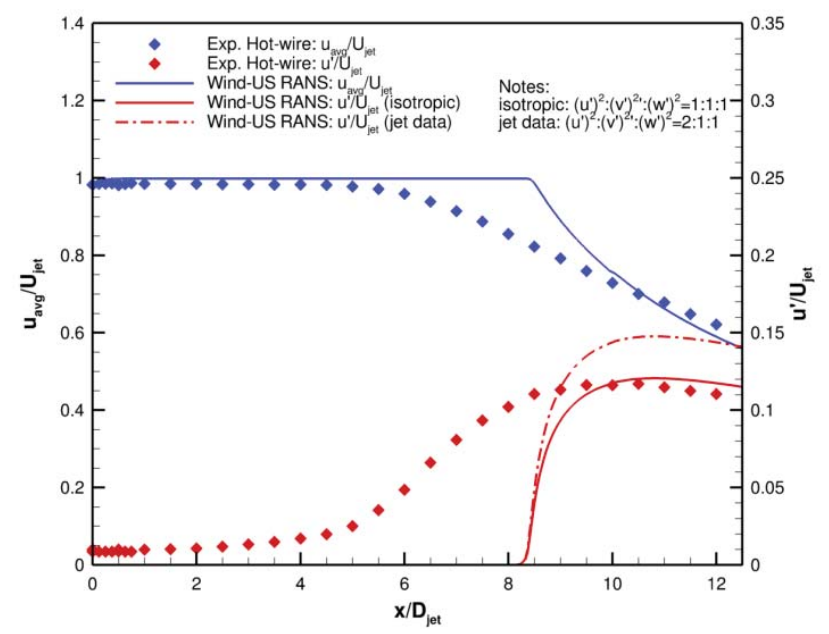

Figure 7: Average velocity (blue) and turbulence intensity (red) along the jet centerline for Mach 1.0T nozzle at $M_{j e t}=0.61$. Results from experimental hot-wire data and RANS simulation are shown. 

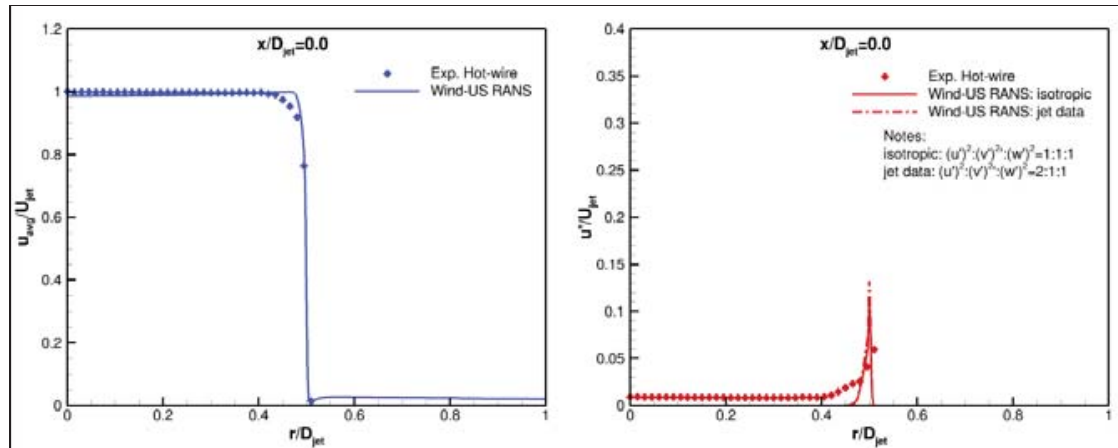

a) $x / D_{j e t}=0.0$
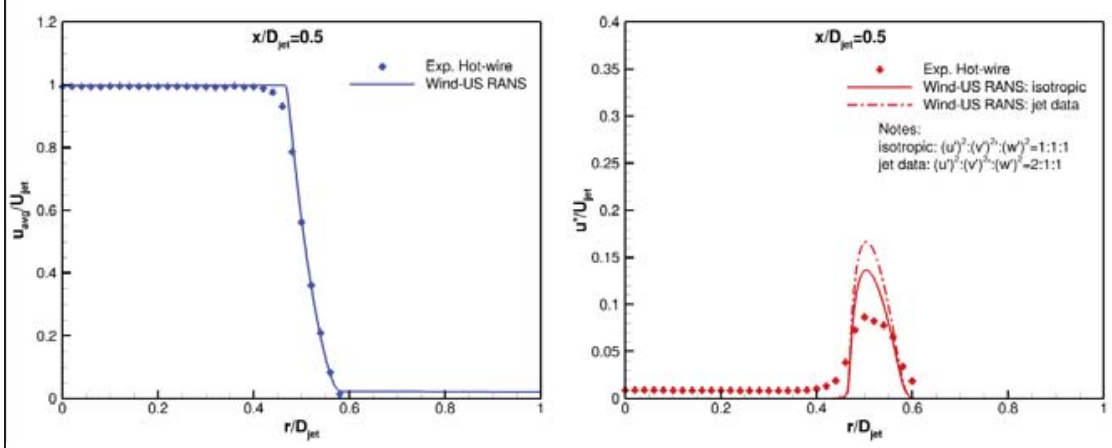

b) $x / D_{j e t}=0.5$
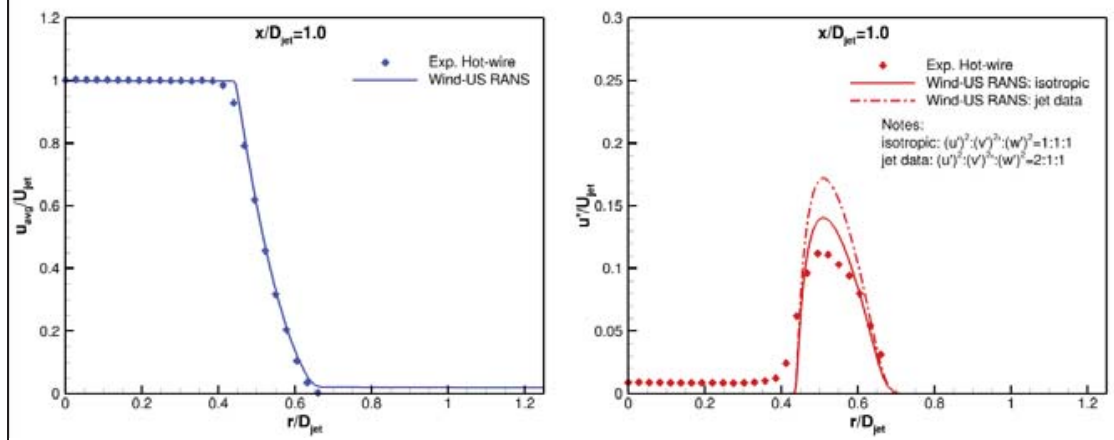

c) $x / D_{\text {jet }}=1.0$

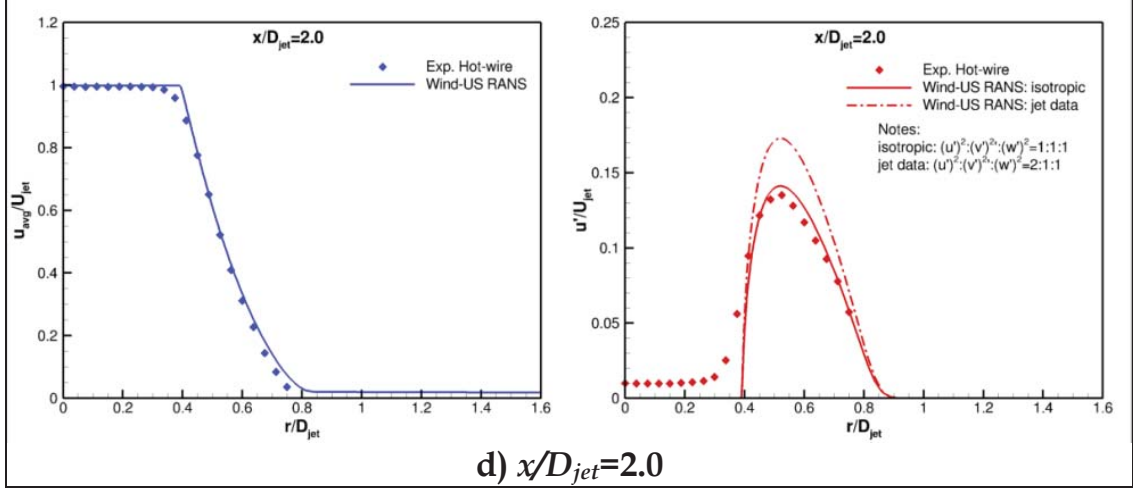

(Figure 8 continues onto the following page.) 

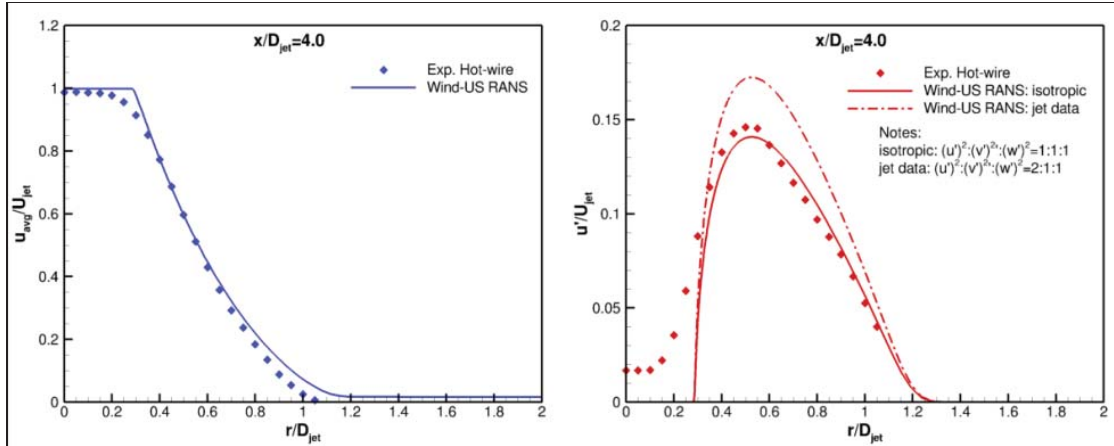

e) $x / D_{\text {jet }}=4.0$
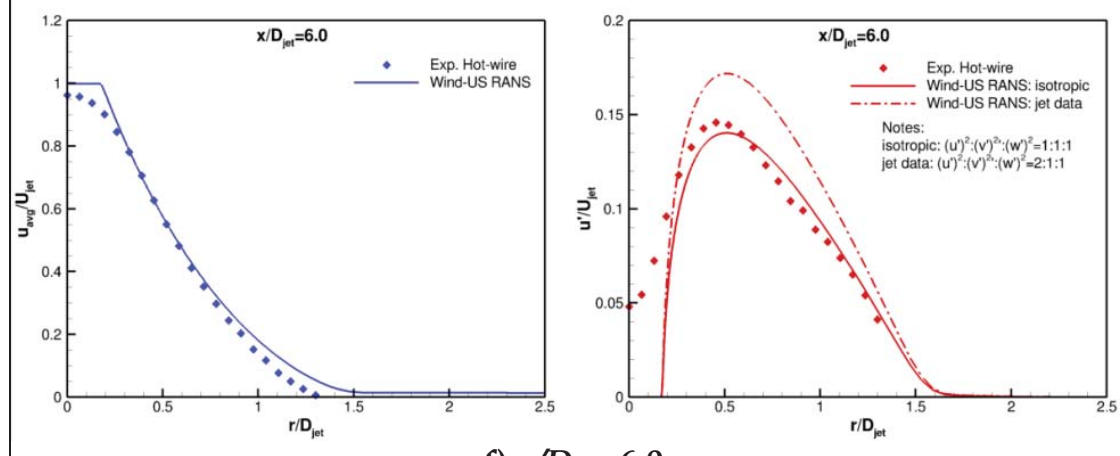

f) $x / D_{\text {jet }}=6.0$
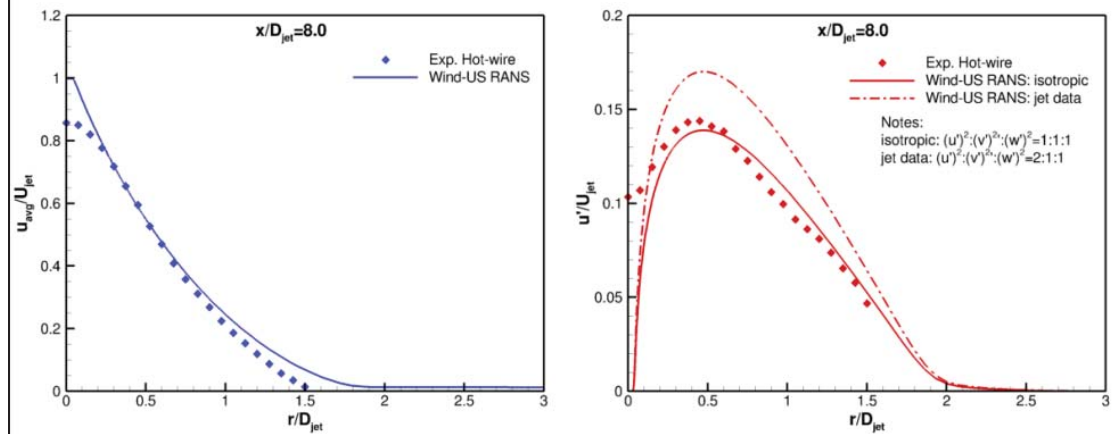

g) $x / D_{\text {jet }}=8.0$
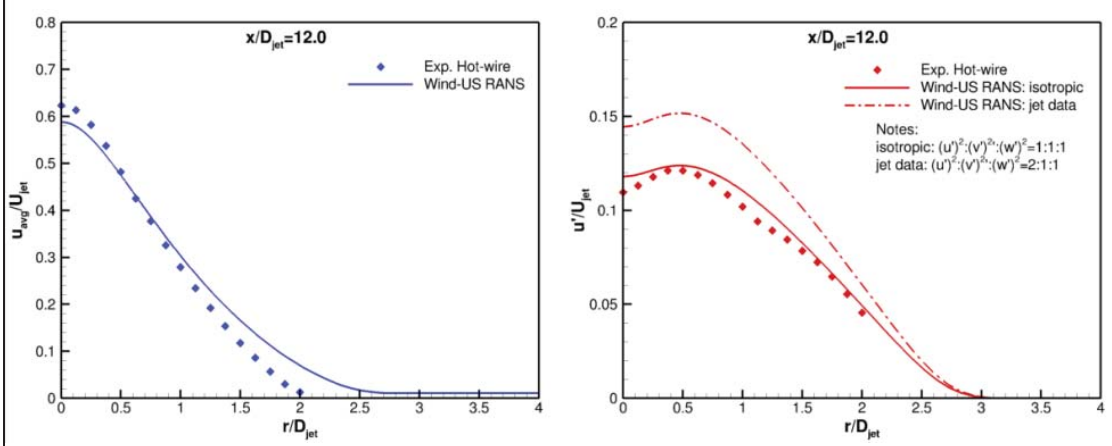

h) $x / D_{\text {jet }}=\mathbf{1 0 . 0}$

Figure 8: Jet plume profiles of average velocity (blue) and turbulence intensity (red) for M10T nozzle at $M_{j e t}=0.61$. Results from experimental hot-wire data and RANS simulation are shown. 


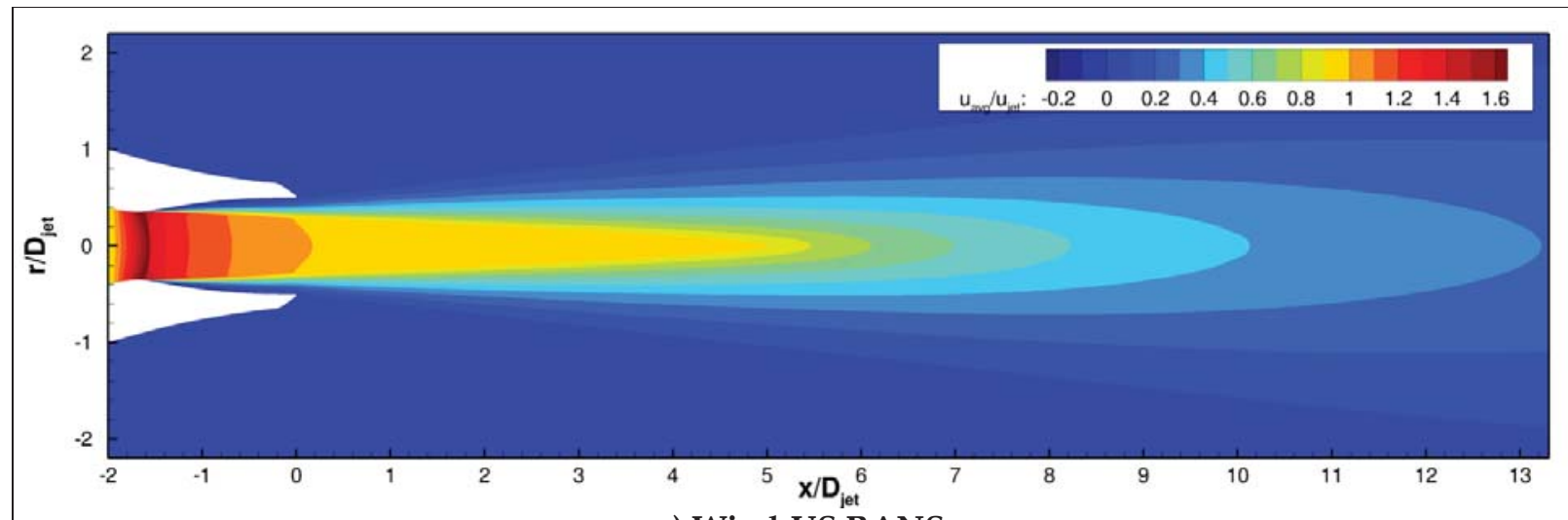

a) Wind-US RANS.

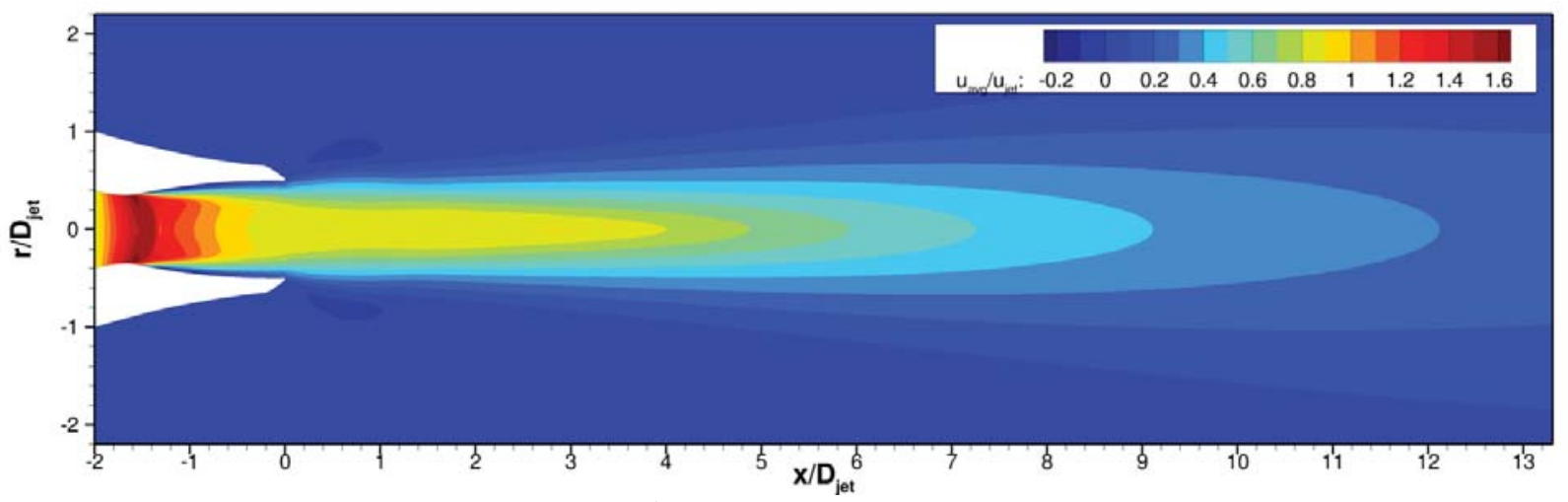

b) Wind-US URANS.

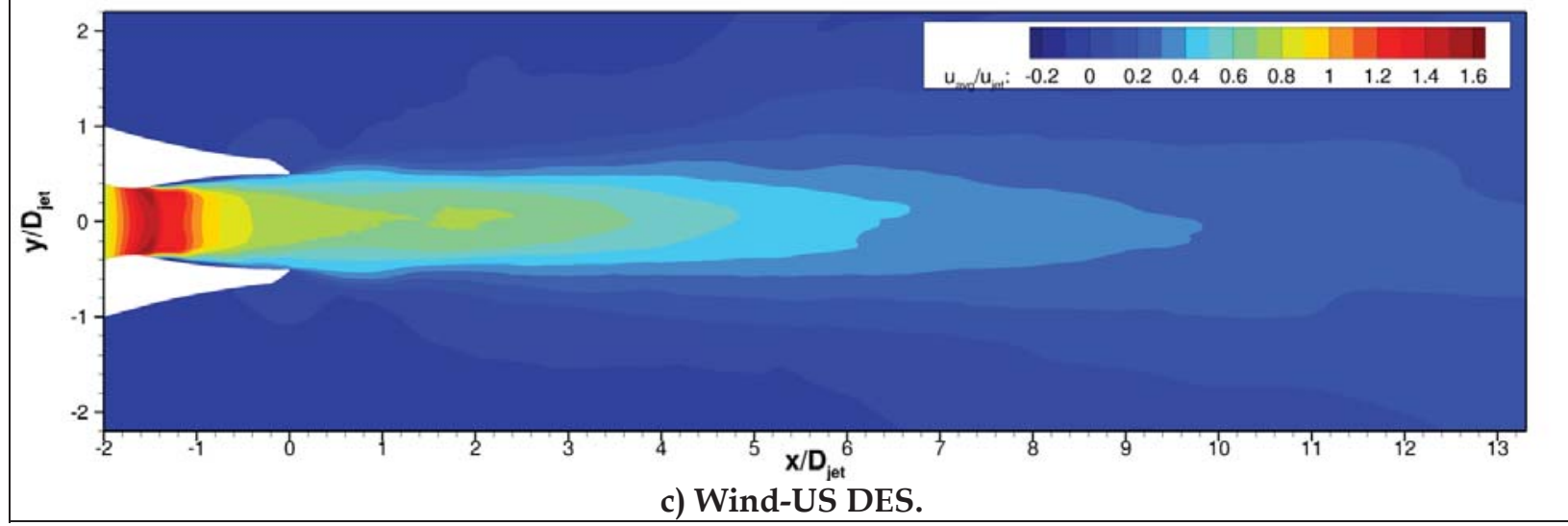

Figure 9: Contours of average velocity of Mach 2.2 nozzle at $M_{j e t}=0.61$. 


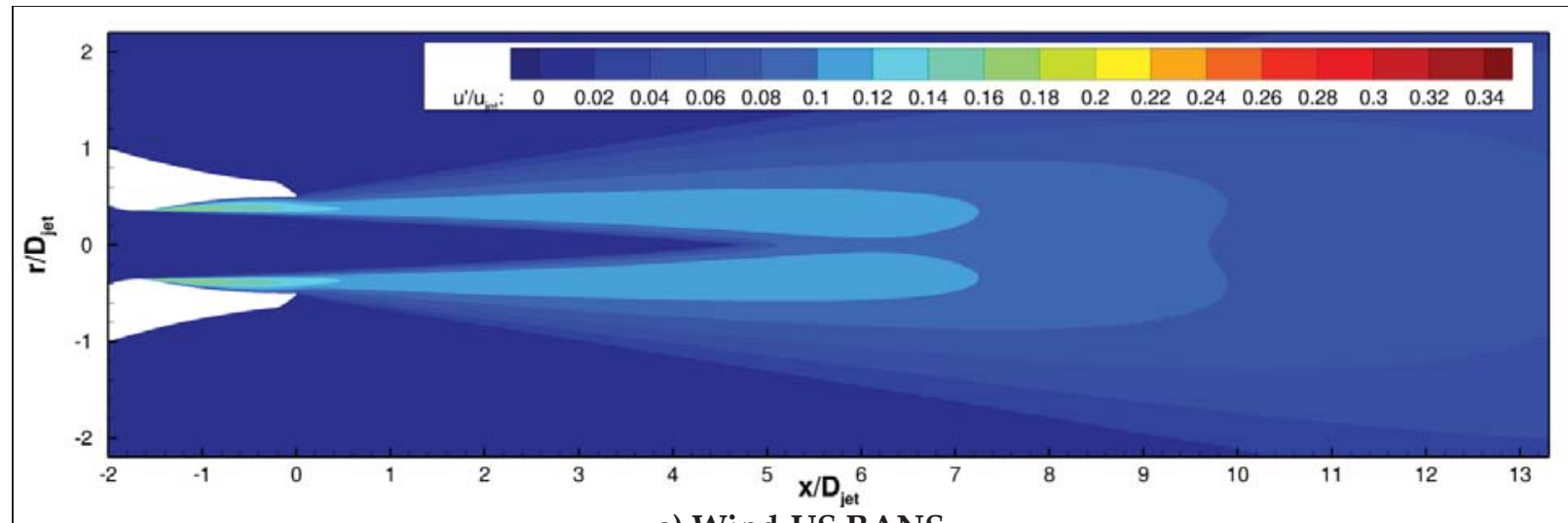

a) Wind-US RANS.

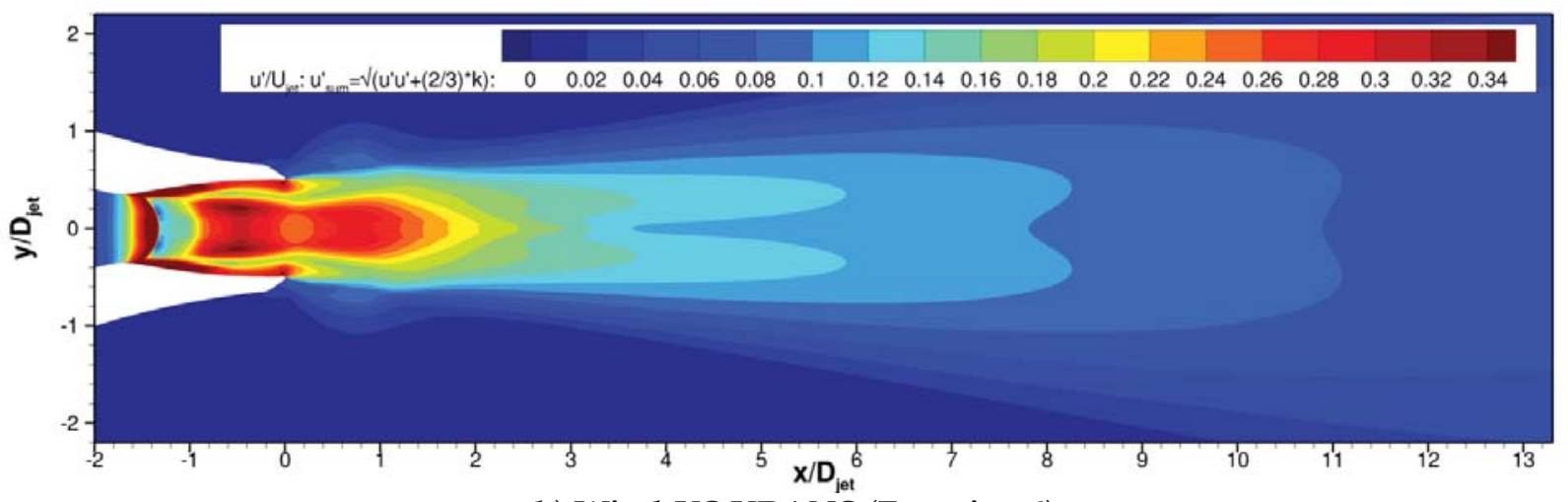

b) Wind-US URANS (Equation 6).

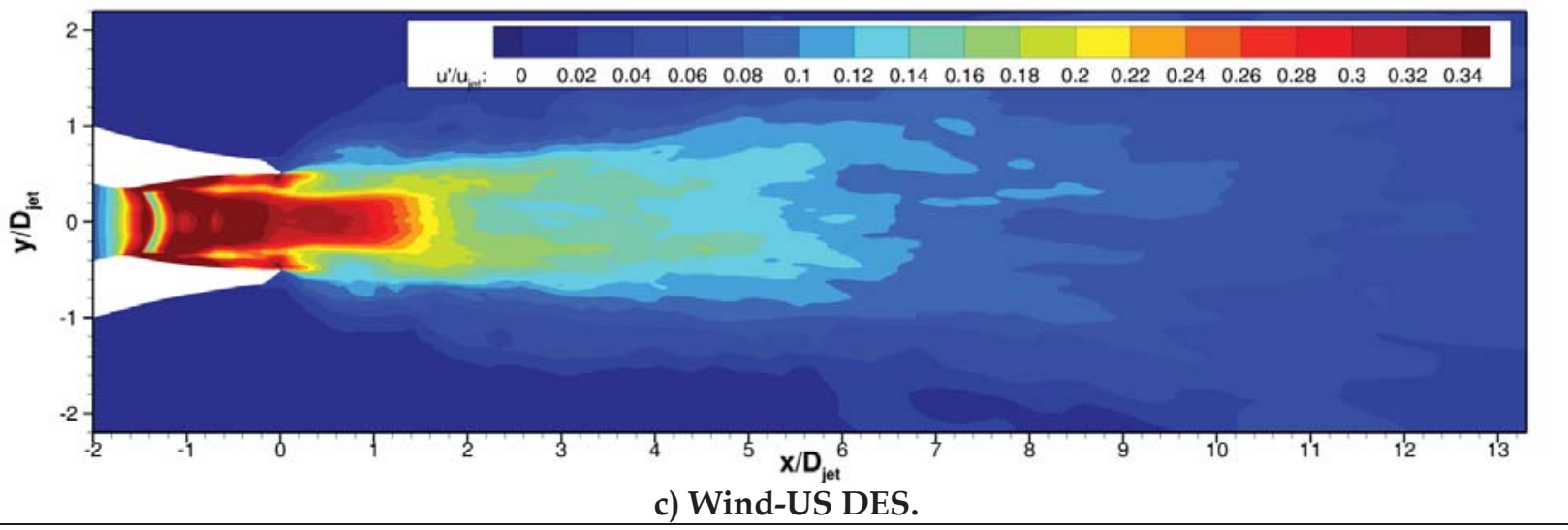

Figure 10: Contours of turbulence intensity of Mach 2.2 nozzle at $M_{j e t}=0.61$. 


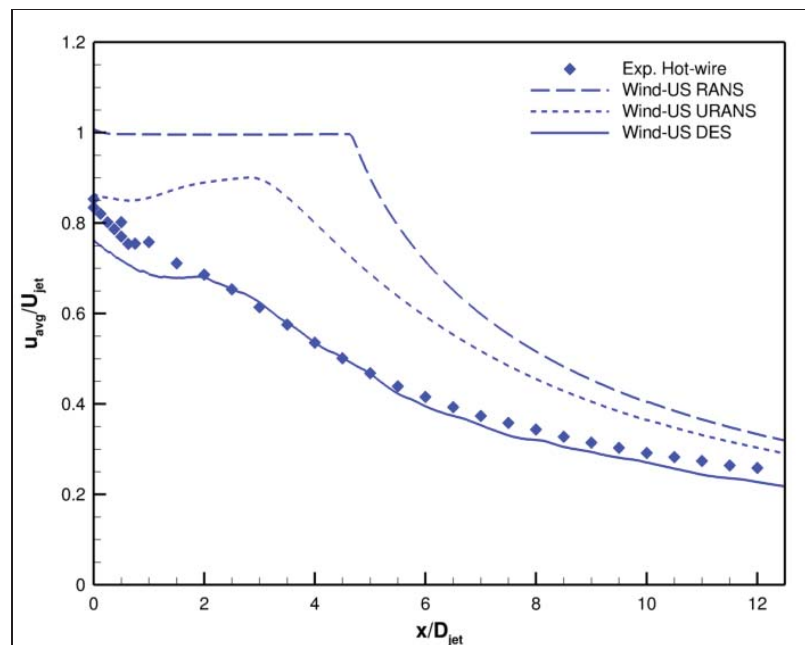

a) Average velocity.

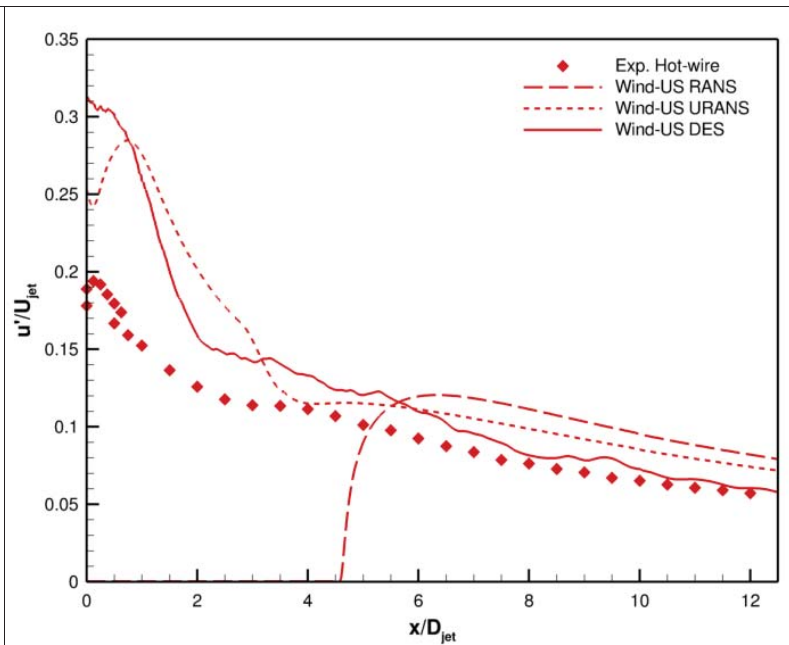

b) Turbulence intensity.

Figure 11: Average velocity (blue) and turbulence intensity (red) along jet centerline for Mach 2.2 nozzle at $M_{j e t}=0.61$. Results from experimental hot-wire data, RANS simulation, URANS simulation, and DES are shown.

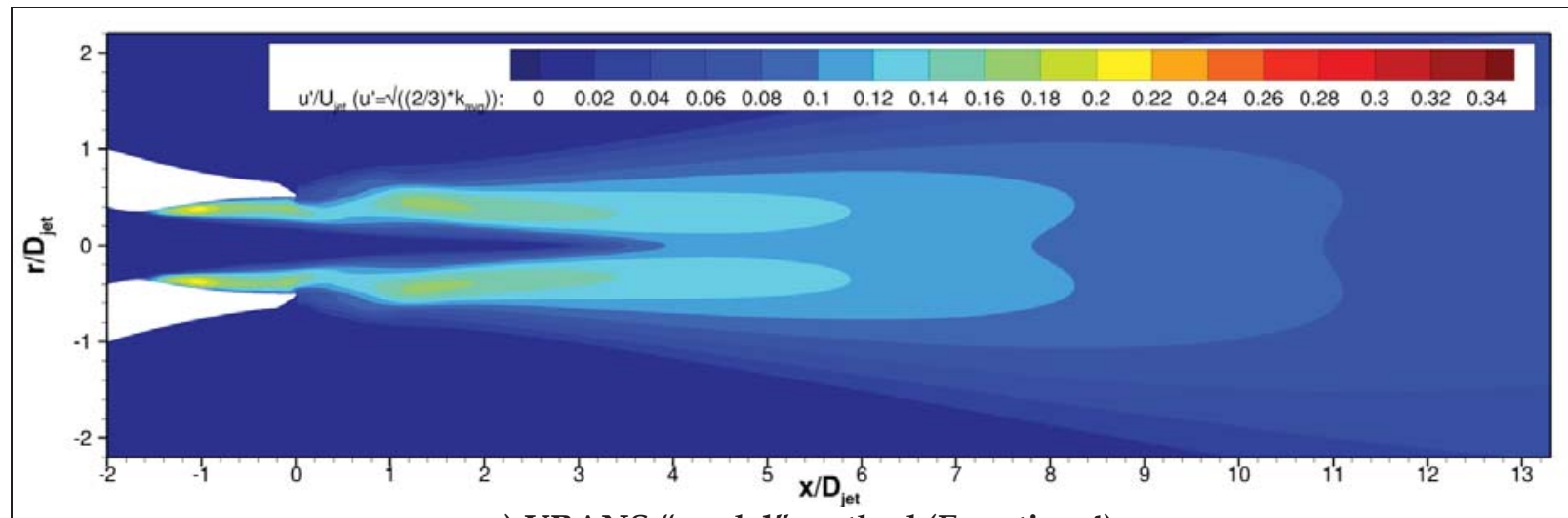

a) URANS "model" method (Equation 4).

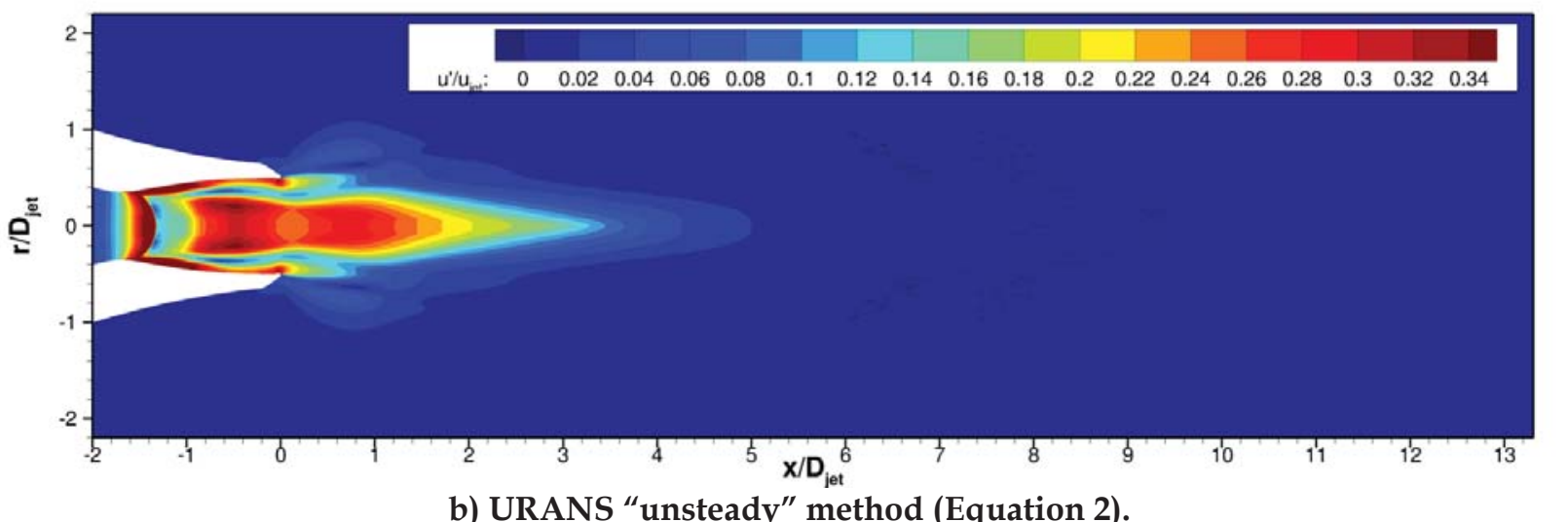

Figure 12: Contours of turbulence intensity of Mach 2.2 nozzle at $M_{j e t}=0.61$ for URANS simulation.

Compare with Figure 9b. 


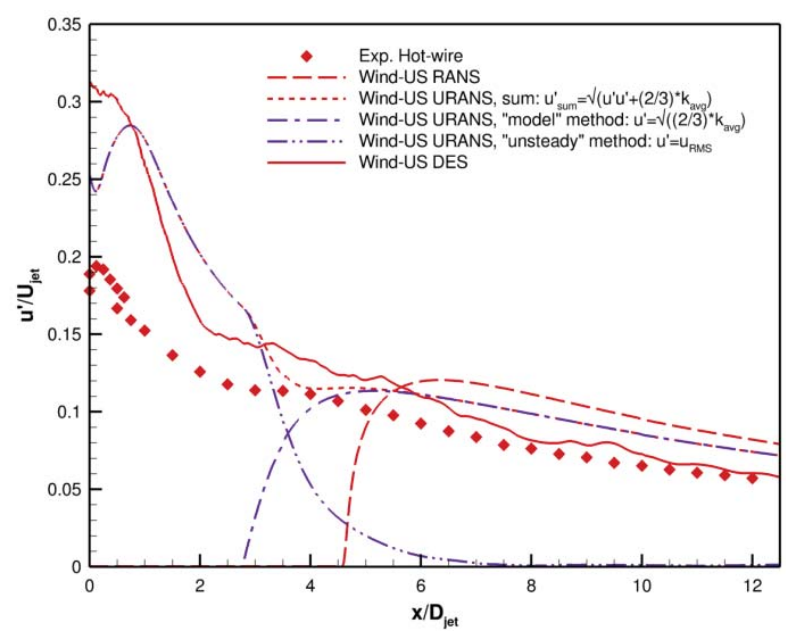

Figure 13: Turbulence intensity along jet centerline for Mach 2.2 nozzle at $M_{j e t}=0.61$. "Model" and "unsteady" methods of computing URANS turbulence intensity (purple lines) are compared with hotwire data and other numerical solutions. 

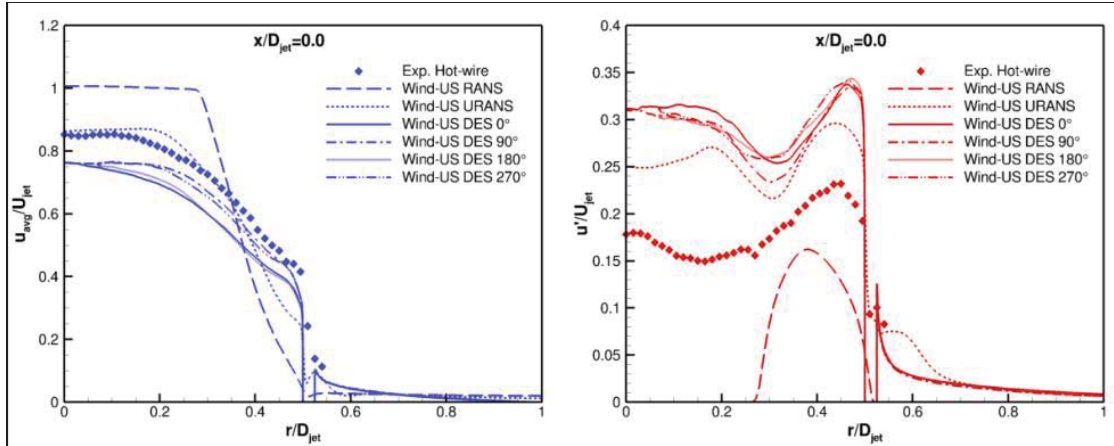

a) $x / D_{\text {jet }}=0.0$
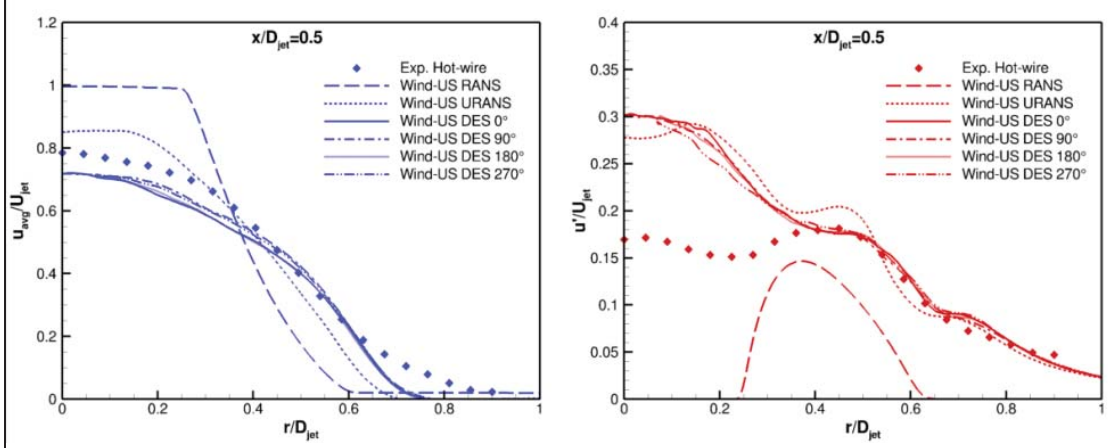

b) $x / D_{\text {jet }}=0.5$
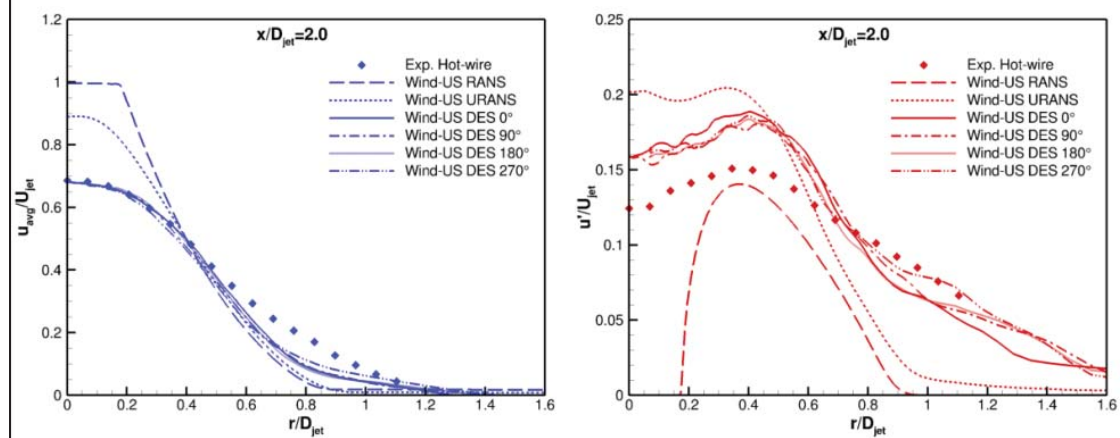

c) $x / D_{j e t}=1.0$
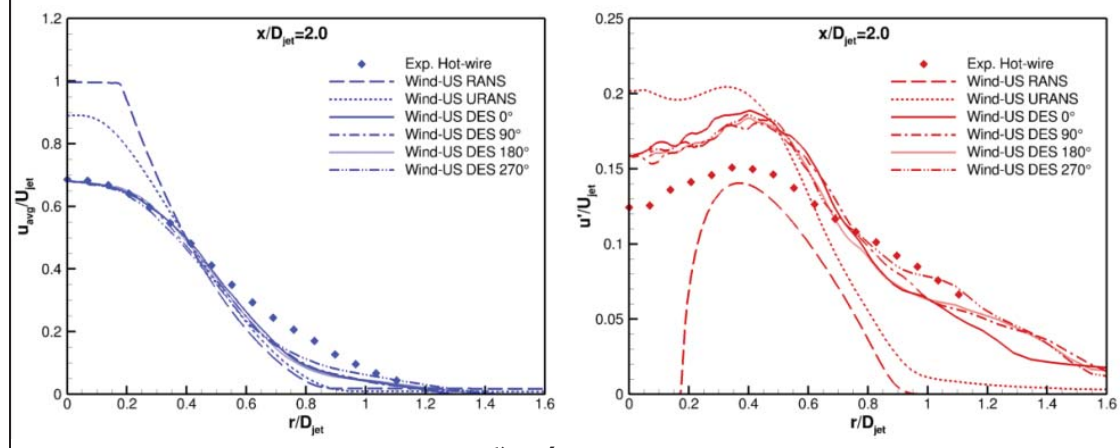

d) $x / D_{\text {jet }}=2.0$

(Figure 14 continues onto the following page.) 

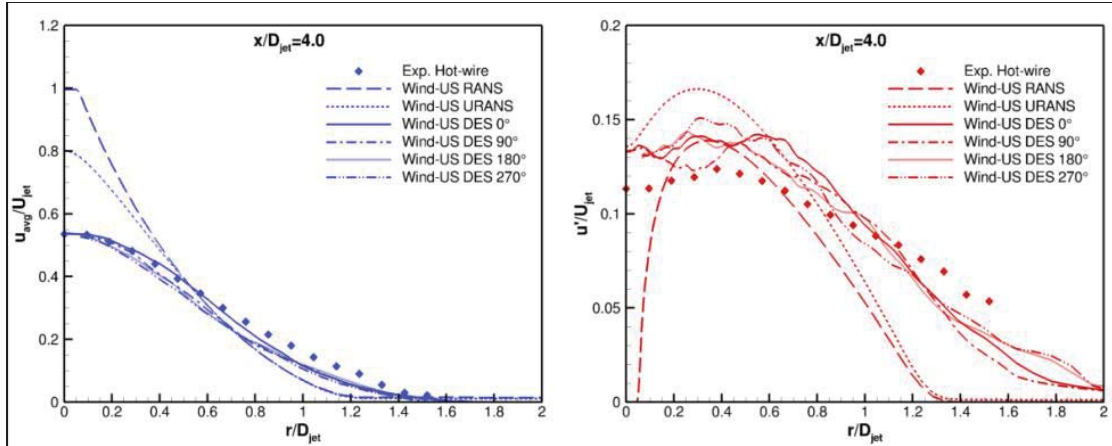

e) $x / D_{j e t}=4.0$
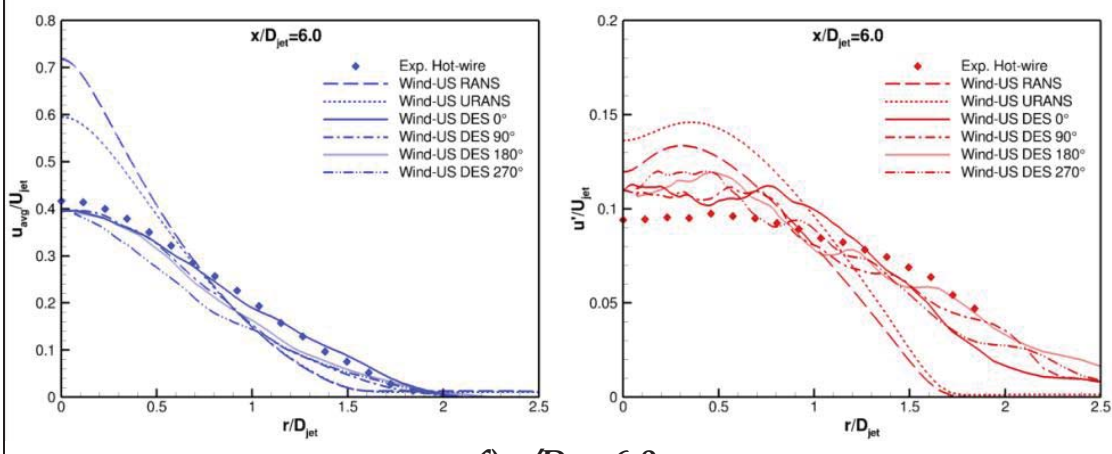

f) $x / D_{j e t}=6.0$
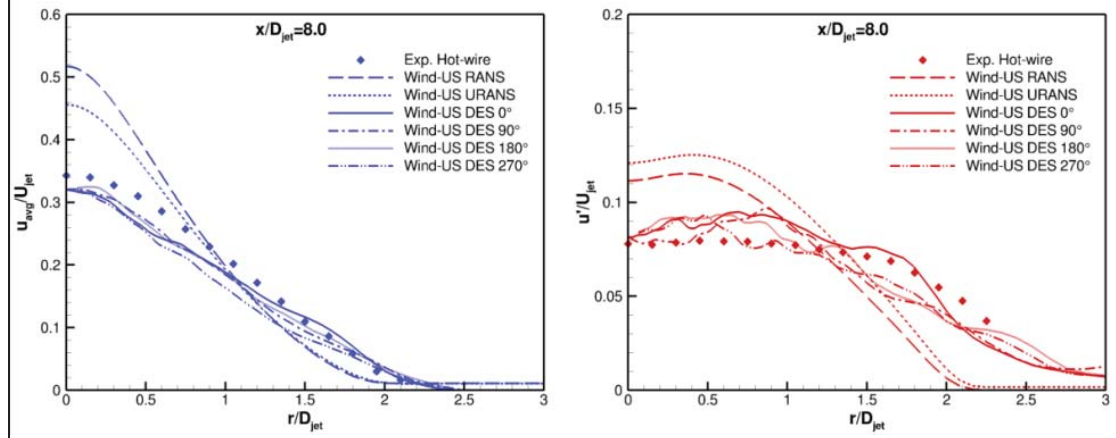

g) $x / D_{\text {jet }}=8.0$
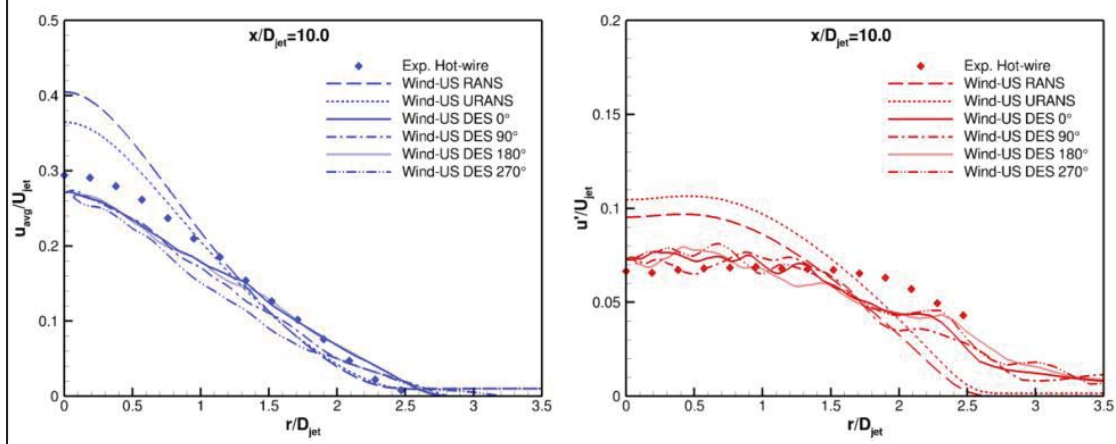

h) $x / D_{\text {jet }}=\mathbf{1 0 . 0}$

Figure 14: Jet plume profiles of average velocity (blue) and turbulence intensity (red) for Mach 2.2 nozzle at $M_{j e t}=0.61$. Results from experimental hot-wire data, RANS simulation, URANS simulation (Equation 6), and DES are shown. 


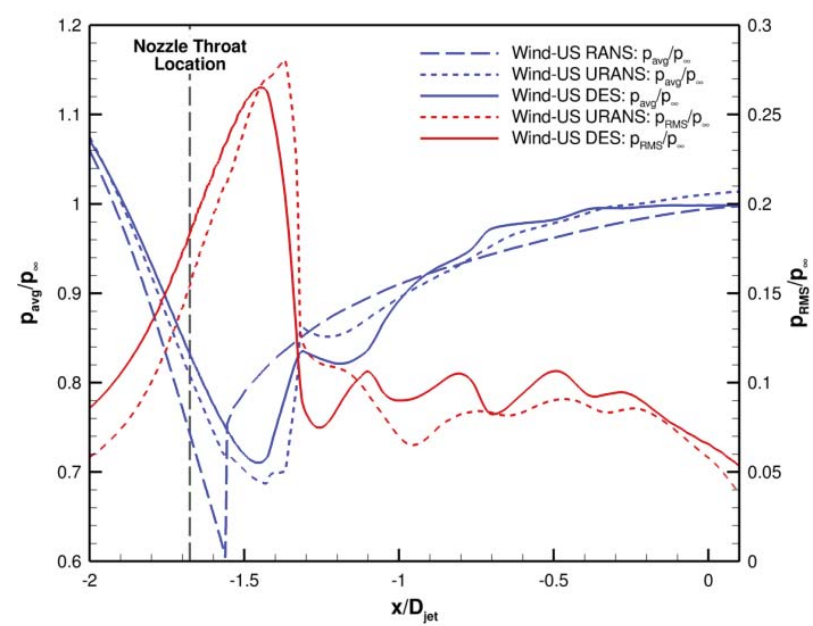

Figure 15: Average pressure (blue) and RMS pressure (red) along centerline inside Mach 2.2 nozzle at $M_{j e t}=0.61$. Results from RANS simulation, URANS simulation, and DES are shown.

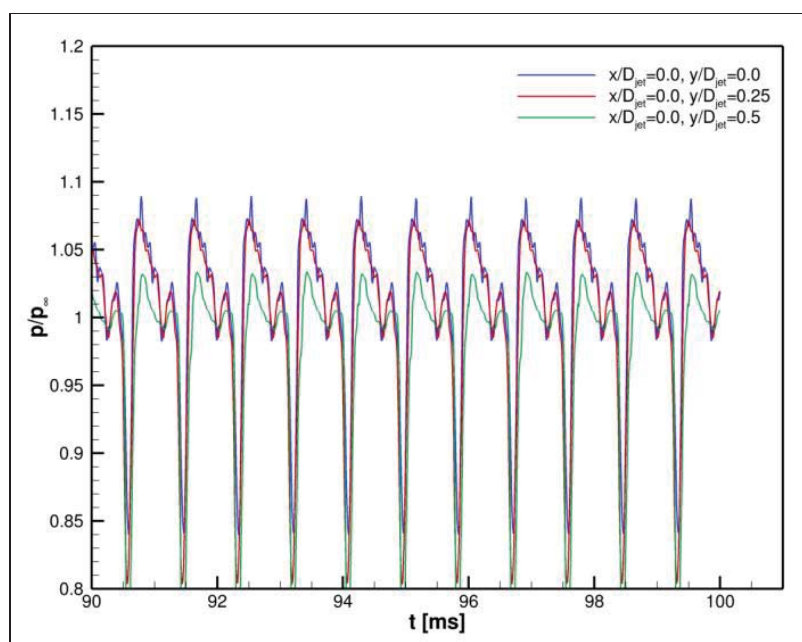

a) URANS

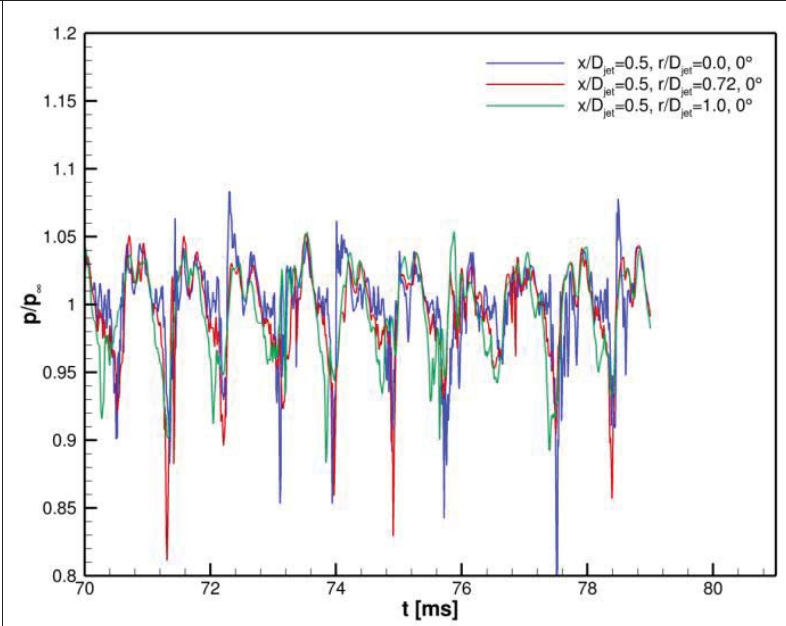

b) DES

Figure 16: Time history of pressure at various probe locations in jet plume of Mach 2.2 nozzle at $M_{j e t}=\mathbf{0 . 6 1}$. 


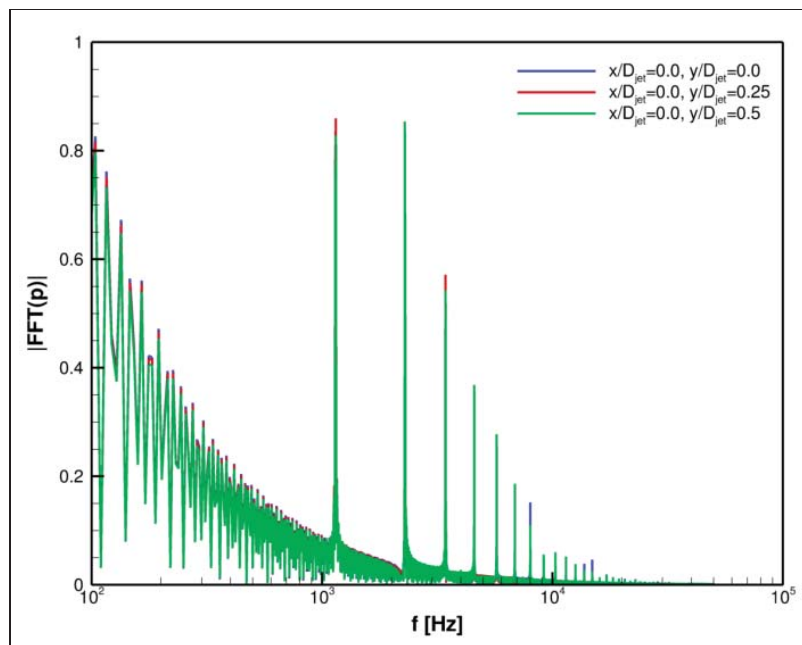

a) URANS

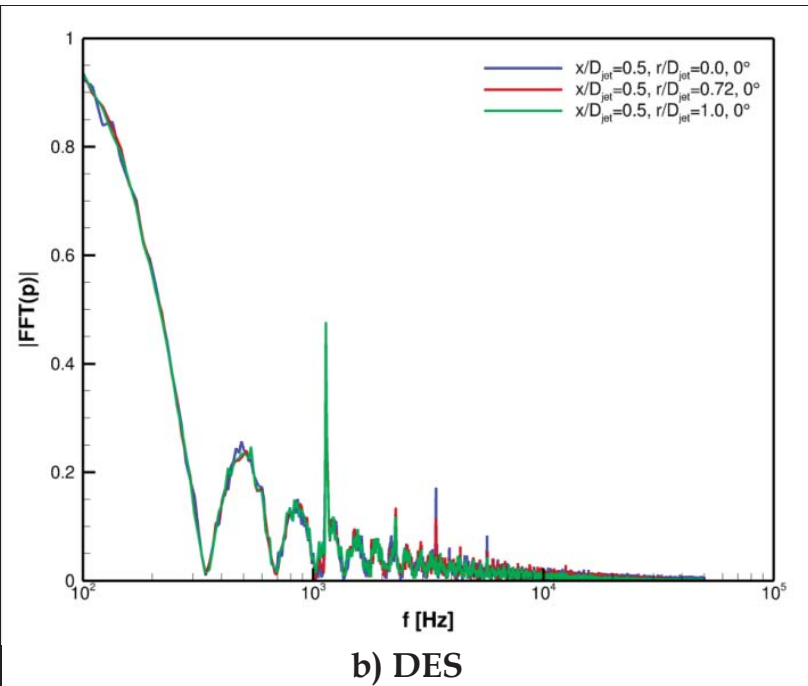

Figure 17: Power Spectral Density of pressure at various probed jet plume locations for Mach 2.2 nozzle at $M_{j e t}=0.61$.

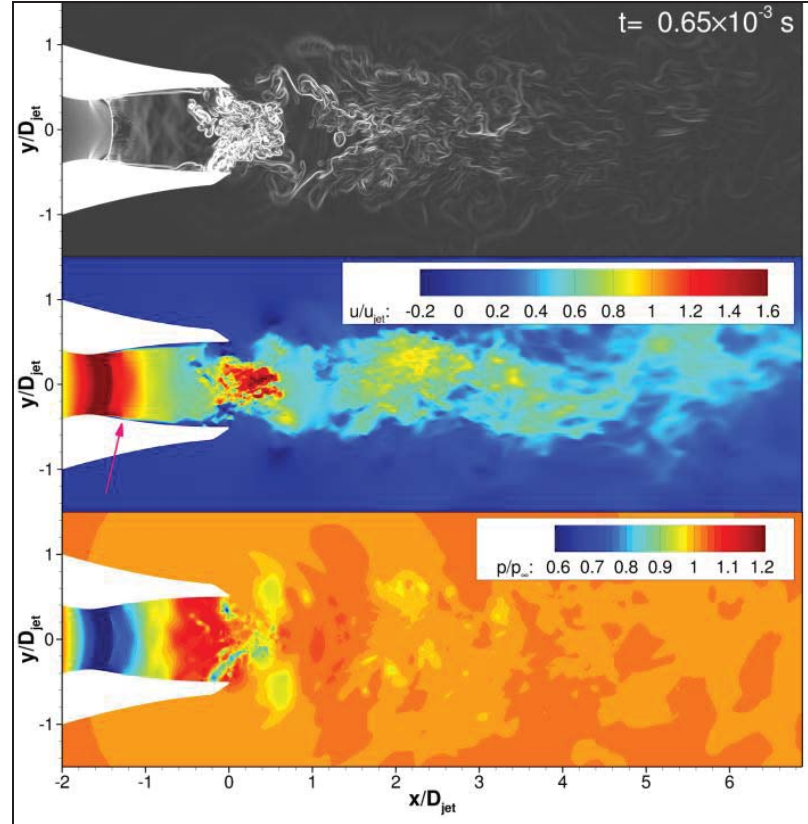

a) Stage 1

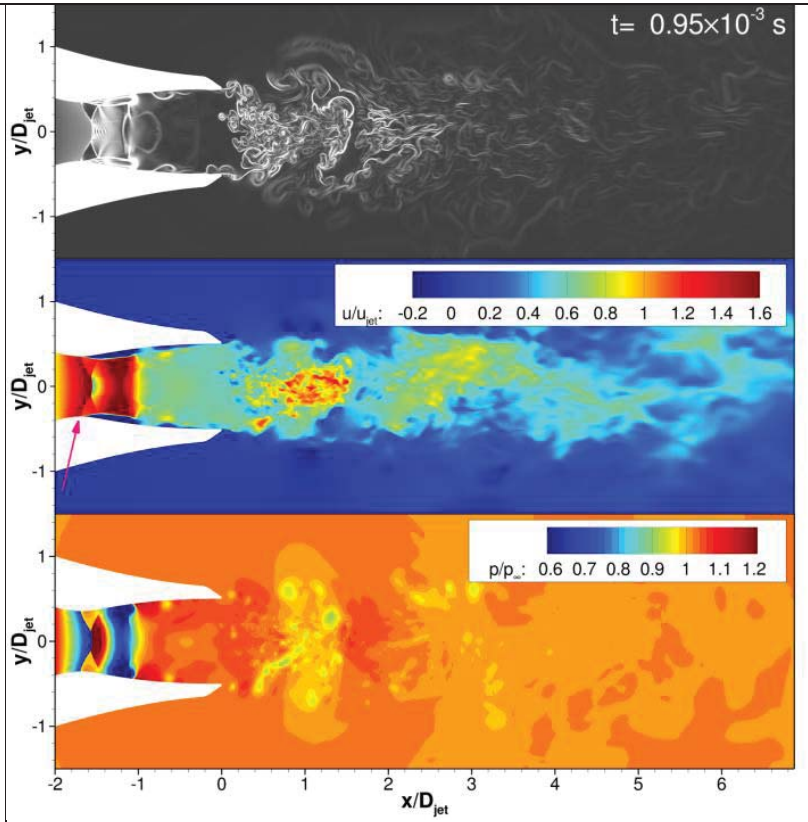

b) Stage 2

(Figure 18 continues onto the following page.) 


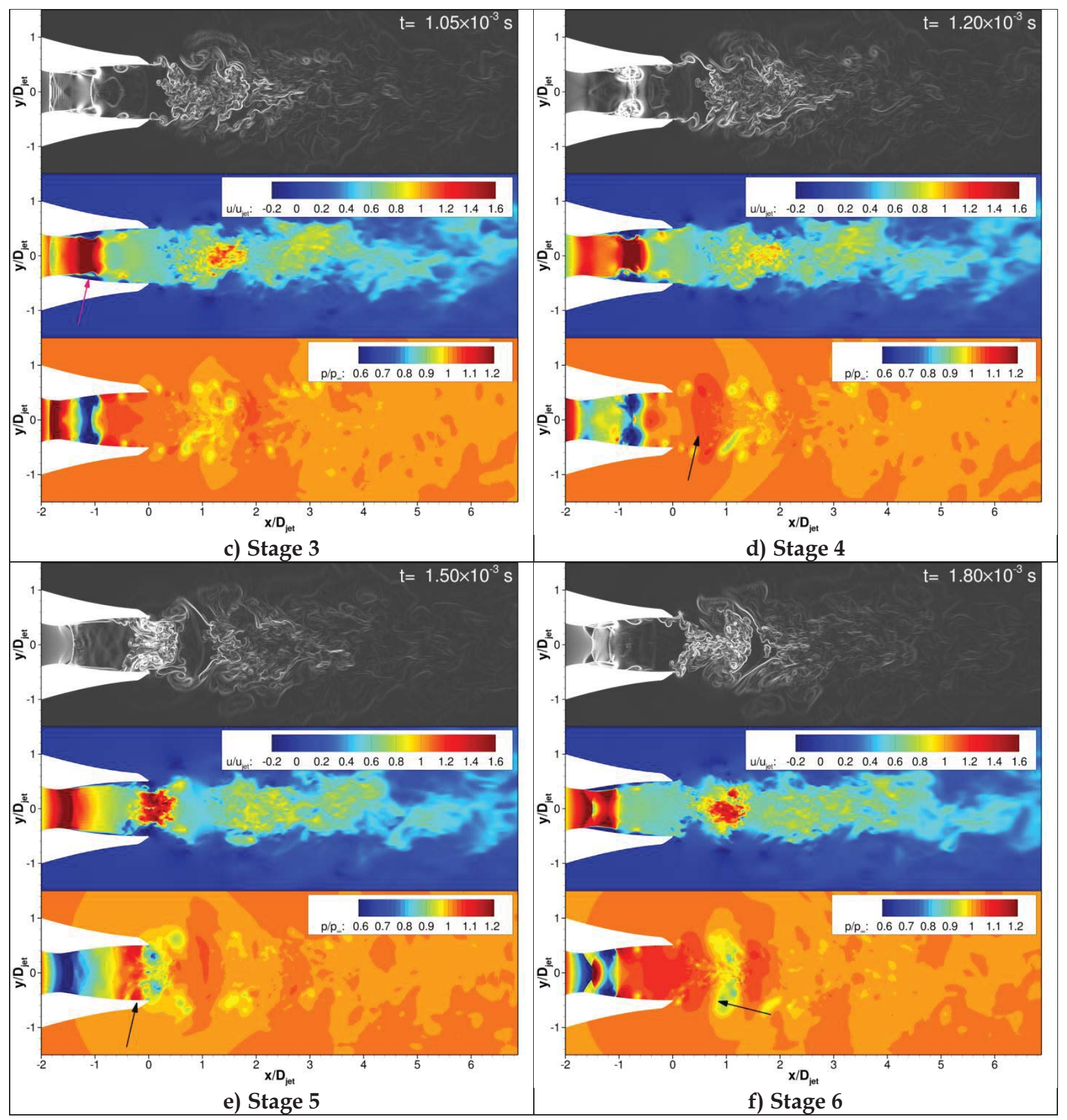

Figure 18: Resonance phenomenon deconstructed for DES of Mach 2.2 nozzle at $M_{j e t}=0.61$. For each time step, contours are shown for instantaneous density gradient (numerical shadowgraph), velocity, and pressure. 